\title{
Running States Estimation of Autonomous Four-Wheel Independent Drive Electric Vehicle by Virtual Longitudinal Force Sensors
}

\author{
Qiu Xia $\mathbb{D}^{1,},{ }^{1,2}$ Long Chen $\mathbb{D}^{\mathbb{D}},{ }^{1,3}$ Xing Xu $\mathbb{D}^{1,},{ }^{1,3}$ Yingfeng Cai $\mathbb{D}^{1,3}$ Haobin Jiang $\mathbb{D},{ }^{1,3}$ \\ Te Chen ${ }^{\mathbb{D}}{ }^{1}$ and Guangxiang Pan $^{2}$
}

${ }^{1}$ School of Automotive and Traffic Engineering, Jiangsu University, Zhenjiang 212013, China

${ }^{2}$ School of Mechanical and Electrical Engineering, Chunzhou University, Chuzhou 239000, China

${ }^{3}$ Automotive Engineering Research Institute, Jiangsu University, Zhenjiang 212013, China

Correspondence should be addressed to Long Chen; chenlong@ujs.edu.cn

Received 10 January 2019; Accepted 21 May 2019; Published 9 June 2019

Academic Editor: Salvatore Strano

Copyright (c) 2019 Qiu Xia et al. This is an open access article distributed under the Creative Commons Attribution License, which permits unrestricted use, distribution, and reproduction in any medium, provided the original work is properly cited.

Exact sideslip angle estimation is significant to the dynamics control of four-wheel independent drive electric vehicles. It is costly and difficult-to-popularize to equip vehicular sensors for real-time sideslip angle measurement; therefore, the reliable sideslip angle estimation method is investigated in this paper. The electric driving wheel model is proposed and applied to the longitudinal force estimation. Considering that electric driving wheel model is a nonlinear model with unknown input, an unknown input estimation method is proposed to facilitate the longitudinal force observer design, in which the adaptive high-order sliding mode observer is designed to achieve the state estimation, the analytic formula of longitudinal force is obtained by decoupling electric driving wheel model, and the longitudinal force estimator is designed by recurrence estimation method. With the designed virtual longitudinal force sensor, an adaptive attenuated Kalman filtering is proposed to estimate the vehicle running state, in which the time-varying attenuation factor is applied to weaken the past data to the current filter and the covariance of process noise and measurement noise can be adjusted adaptively. Finally, simulations and experiments are conducted and the effectiveness of proposed estimation method is validated.

\section{Introduction}

Electric vehicles have been extensively recognized as promising vehicle architectures with several advantages like high capacity usage ratio, more simplified transmission structure and environmental friendly characteristics $[1,2]$. Fourwheel independent drive electric vehicles (FWID-EVs), in virtue of precise and flexible torque control, have been admitted feasible and effective to improve vehicle stability. Many advanced active safety control systems have been widely used for ground vehicles to prevent fatal accidents and guarantee safety $[3,4]$. Active safety system, of which the antilock brake system, the electronic stability program, the traction control system, and adaptive cruise control, collision warning are examples, is significant to vehicle stability control during complicated conditions and severe maneuvers. The sideslip angle of ground vehicles is defined as the angle between the vehicle longitudinal axis and the vehicle velocity vector. Lots of vehicle motion control systems need to monitor the sideslip angle dynamically, that is to say, the stable performance of vehicle motion control is dependent on precise and credible sideslip angle information [5-9]. Recently, the auxiliary driving and self-driving, with the advantage of vehicle autonomous security and reduced mobility cost, has attracted views and considerate efforts from both researchers and companies [10-12], and the estimation of sideslip angle is also important to the vehicle pathfollowing and lateral stability control $[13,14]$. However, 
the sideslip angle is hard and costly to obtain directly by vehicular sensors. Based on this consideration, it is essential to research the design of accurate and stable sideslip angle observer.

With the development and deepening of relevant research in vehicle running states estimation, many universities and automobile enterprises have made full research on this issue, achieving some great research results. The estimation means used for vehicle running states estimation in prior studies includes Kalman-filter-based method [15-22], nonlinearestimator-based method [23-28], error iteration method [2931 , redundancy measurement amalgamation method [3237], and antijamming estimation [38]. Jin proposed a lateral tire forces and sideslip angle estimation method based on unscented Kalman filter with the consideration of the nonlinear tire model and using the interacting multiple model filter estimation method to improve the estimation accuracy [16]. Cheng designed a novel vehicle sideslip angle fusion estimation method using the adaptive square-root cubature Kalman filter, in which a weight coefficient was introduced to improve the adaptive ability of the designed Kalman filter [17]. With the research of vehicle state estimation methods tending to meet the practical needs of engineering applications, people gradually tend to select the corresponding estimation algorithms according to the causes of the estimation errors of the research objects to obtain better estimation results. To guarantee the yaw-stability and roll-stability control of vehicle, Nam presented a sideslip angle and roll angle estimation method using the extended Kalman filter, with the lateral tire force being directly measured by equipped vehicular sensors [33]. In addition, some researchers recently begin to study the robust sideslip angle observer design and achieve many great research results $[39,40]$, in which the uncertainty of vehicle dynamic system caused by parameter perturbation is considered and used for parametric vehicle uncertainty modelling. Zhang established an uncertain linear parameter varying vehicle model with consideration of the variation of longitudinal velocity and proposed a gain-scheduling observer for sideslip angle estimation [40]. The electric driving wheel model (EDWM), consisting of in-wheel motors and wheels, is not only an independent control unit, but also an independent information unit. The measurements of current, voltage, and wheel speed of EDWM are easily to be obtained by low-cost sensors. If we apply this feature to vehicle state estimation method, it will help to make full use of the electric driving advantage of FWID-EVs, improve the estimation accuracy, and reduce the estimation cost. In $[6,41]$, the electric-drive characteristic of in-wheel motor drive electric vehicle is introduced into the estimation of longitudinal tire force. In the above research literatures, the longitudinal force is estimated by designing the unknown input observer, and the system states are used as the measurements directly. Compared with above research literatures, in this paper, a sliding mode observer is designed in advance to estimate the system state, and the system state estimation results are then used as the virtual measurements in the longitudinal force estimation. Thus, the estimation accuracy can be further improved. Moreover, an adaptive attenuated Kalman filter (AAKF) is studied for vehicle running state estimation.
In this work, motivated by above analysis, a running states estimation method of autonomous four-wheel independent drive electric vehicle by virtual longitudinal force sensors (VLFS) is presented. Making full use of the electric driving characteristics of FWID-EV, the EDWM is proposed and introduced to the longitudinal force estimation. Considering the EDWM is a nonlinear model with unknown input, an unknown input estimation method is proposed to facilitate the longitudinal force observer design. The designed longitudinal force observer is seen as a VLFS and used to provide the pseudo-measurement input to the vehicle running state estimation. The AAKF is proposed to estimate the vehicle running state, in which the time-varying attenuation factor is applied to weaken the past data to the current filter and the covariance of process noise and measurement noise can be adjusted adaptively.

The rest of this paper is organized as follows. The vehicle dynamics model is presented in Section 2. The vehicle sideslip angle estimation method is designed in Section 3. The simulation results are provided in Section 4. The experimental results are shown in Section 5, followed by the conclusive remarks.

\section{Vehicle Dynamics Model}

2.1. 3-DOF Vehicle Model. Vehicle dynamic behavior modeling is the basis of vehicle state observer design. In this paper, the pitch, roll motion, and mechanical characteristics of four driving wheels are neglected, and only the degree of freedom of vehicle in longitudinal and lateral directions and yaw directions are considered to establish the vehicle dynamic relationship. The force analysis of the vehicle is shown in Figure 1. The center of mass of the vehicle is regarded as the origin of the driving coordinate system. The $x$-axis is directed to the driving direction of the vehicle, the $y$-axis is directed to the left of the driver, and the yaw direction is directed counterclockwise around the origin of the coordinate system. The numbers $1,2,3$, and 4 of the wheel represent the frontleft, the front-right, the rear-left, and the rear-right wheel, respectively. The dynamic equations of the 3-DOF vehicle model can be written as

$$
\begin{aligned}
\dot{v}_{x} & =\gamma v_{y}+\frac{1}{m}\left[\left(F_{x 1}+F_{x 2}\right) \cos \delta-\left(F_{y 1}+F_{y 2}\right) \sin \delta\right. \\
& \left.+F_{x 3}+F_{x 4}\right] \\
\dot{v}_{y} & =-\gamma v_{x}+\frac{1}{m}\left[\left(F_{x 1}+F_{x 2}\right) \sin \delta+\left(F_{y 1}+F_{y 2}\right) \cos \delta\right. \\
& \left.+F_{y 3}+F_{y 4}\right] \\
\dot{\gamma} & =\frac{1}{I_{z}}\left[\left(F_{x 1}+F_{x 2}\right) l_{f} \sin \delta-\left(F_{y 3}+F_{y 4}\right) l_{r}\right. \\
& +\left(F_{y 1}+F_{y 2}\right) l_{f} \cos \delta+\left(F_{y 1}-F_{y 2}\right) b_{f} \sin \delta \\
& \left.-\left(F_{x 1}-F_{x 2}\right) b_{f} \cos \delta-\left(F_{x 3}-F_{x 4}\right) b_{r}\right]
\end{aligned}
$$

where $v_{x}$ and $v_{y}$ represent the longitudinal and lateral vehicle speed, respectively. $\gamma$ represents the yaw rate, $\beta$ represents the vehicle sideslip angle, $m$ is the vehicle mass, $\delta$ represents 


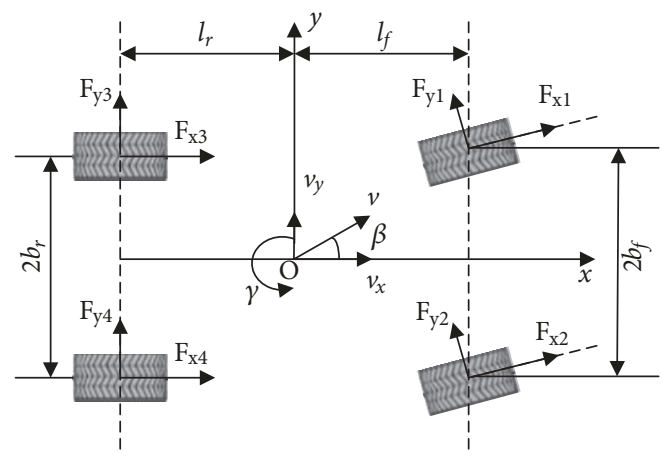

Figure 1: Vehicle dynamics model.

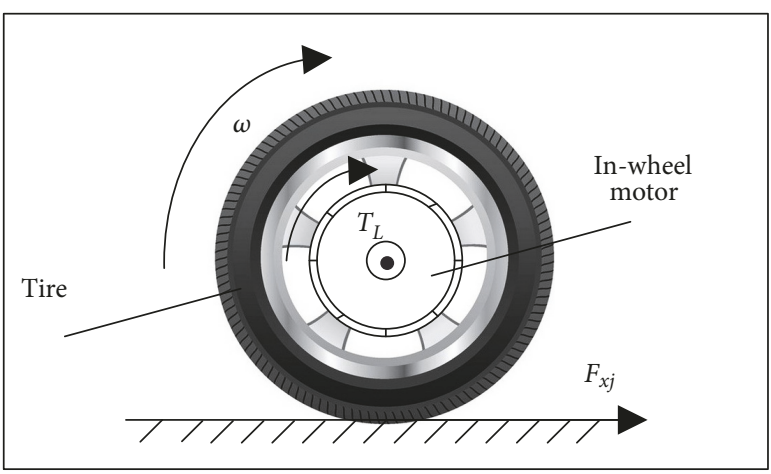

FIGURE 2: Electric driving wheel model.

the front-wheel steering angle, $I_{z}$ stands for the moment of inertia. $F_{x j}$ and $F_{y j}(j=1,2,3,4)$ are the longitudinal tire force and lateral tire forces, respectively. $l_{f}$ and $l_{r}$ denote the front-axle distance and the rear-axle distance, respectively. $b_{f}$ and $b_{r}$ denote the front-half wheelbase and rear-half wheelbase, respectively.

2.2. EDWM. The diagrammatic sketch of EDWM is shown in Figure 2. The FWID-EV is actuated by four in-wheel motors, and the electromechanical coupling driving wheel composed a motor and a tire can be considered as an independent information module, so the concept of the EDMW is introduced to the longitudinal force estimation process and the current, speed, and voltage are used to estimate the longitudinal force. The state equation of EDWM is expressed as

$$
J_{1} \dot{\omega}_{j}=T_{L j}-F_{x j} r
$$

where $\omega_{j}$ represents the wheel rotational speed, $J_{1}$ denotes the inertia moment, $r$ represents the effective rolling radius of EDWM, and $T_{L j}$ stands for the motor load torque. The output-axis torque dynamics equation of EDWM is shown as

$$
J_{2} \dot{\omega}_{j}+b \omega_{j}=K_{t} i_{j}-T_{L j}
$$

where $J_{2}$ is the motor-rotor rotation inertia, $b$ represents the damping coefficient, $K_{\mathrm{t}}$ is the torque constant of in-wheel motor, and $i_{j}$ denotes the line current. The equivalent-circuit voltage equation of EDWM is given by

$$
u_{j}=R i_{j}+L \dot{i}_{j}+K_{a} \omega_{j}
$$

where $u_{j}$ denotes the line voltage of in-wheel motor, $R$ denotes the equivalent resistance, $L$ denotes the equivalent inductance, and $K_{a}$ denotes the back electromotive force coefficient.

2.3. Tire Model. Accurate tire force model is the basis of vehicle driving state estimation. The tire model of magic formula is computed as

$$
F_{x, y}=D \sin \{C \arctan [B \alpha-E(B \alpha-\arctan (B \alpha))]\} .
$$

where $B$ denotes the stiffness factor, $C$ denotes the curveshape factor, $D$ denotes the peak factor, and $E$ denotes the curve curvature factor, $\alpha$ denotes the sideslip angle of each wheel. The $B, C, D, E$ in (7) vary with the change of the tire vertical tire, and the tire vertical force is obtained as

$$
\begin{aligned}
& F_{z 1}=l_{r}\left(\frac{m g}{2 l}+\frac{m a_{y} h}{2 b_{f} l}\right)-\frac{m a_{x} h}{2 l} \\
& F_{z 2}=l_{r}\left(\frac{m g}{2 l}-\frac{m a_{y} h}{2 b_{f} l}\right)-\frac{m a_{x} h}{2 l} \\
& F_{z 3}=l_{f}\left(\frac{m g}{2}+\frac{m a_{y} h}{2 b_{r} l}\right)+\frac{m a_{x} h}{2 l} \\
& F_{z 4}=l_{f}\left(\frac{m g}{2}-\frac{m a_{y} h}{2 b_{r} l}\right)+\frac{m a_{x} h}{2 l} .
\end{aligned}
$$

where $F_{z 1}, F_{z 2}, F_{z 3}$, and $F_{z 4}$ are the vertical tire forces, $h$ denotes the gravity-center height to the horizontal plane, and $g$ is the gravity acceleration. The wheel sideslip angle is written as

$$
\begin{aligned}
& \alpha_{1}=\delta-\arctan \frac{v_{y}+l_{f} \gamma}{v_{x}+b_{f} \gamma / 2} \\
& \alpha_{2}=\delta-\arctan \frac{v_{y}+l_{f} \gamma}{v_{x}-b_{f} \gamma / 2} \\
& \alpha_{3}=-\arctan \frac{v_{y}-l_{r} \gamma}{v_{x}+b_{r} \gamma / 2} \\
& \alpha_{4}=-\arctan \frac{v_{y}-l_{r} \gamma}{v_{x}-b_{r} \gamma / 2} .
\end{aligned}
$$

\section{Vehicle Sideslip Angle Estimation Method}

3.1. The Design of VLFS. Substituting formula (4) into formula (5) and coupling it with formula (6), we can obtain the EDWM as follows:

$$
\begin{aligned}
& \dot{i_{j}}=-\frac{R}{L} i_{j}-\frac{K_{a}}{L} \omega_{j}+\frac{1}{L} u_{j} \\
& \dot{\omega}_{j}=\frac{K_{t}}{J} i_{j}-\frac{b}{J} \omega_{j}-\frac{r}{J} F_{x j}
\end{aligned}
$$


where $J=J_{1}+J_{2}$. The EDWM in formula (9) can be written as

$$
\begin{aligned}
& \dot{x}_{f}=A_{f} x_{f}+B_{f} u_{f}+D_{f} d_{f} \\
& y_{f}=C_{f} x_{f}
\end{aligned}
$$

where $x_{f}$ is the system state, $u_{f}$ is the system input, $d_{f}$ is the system unknown input, and $y_{f}$ is the system measurement output, respectively. The physical meaning of known input is motor voltage, and the physical meaning of unknown input is longitudinal tire force. And,

$$
\begin{aligned}
x_{f} & =\left[\begin{array}{ll}
i_{j} & \omega_{j}
\end{array}\right]^{T}, \\
A_{f} & =\left[\begin{array}{rr}
-\frac{R}{L} & -\frac{K_{a}}{L} \\
\frac{K_{t}}{J} & -\frac{b}{J}
\end{array}\right], \\
B_{f} & =\left[\begin{array}{c}
\frac{1}{L} \\
0
\end{array}\right], \\
D_{f} & =\left[\begin{array}{c}
0 \\
-\frac{r}{J}
\end{array}\right], \\
C_{f} & =\left[\begin{array}{ll}
1 & 0 \\
0 & 1
\end{array}\right] .
\end{aligned}
$$

The virtual longitudinal force sensor is actually an artificially designed longitudinal force observer, and the estimated value of longitudinal force observer is regarded as the pseudomeasurement of longitudinal force sensor and used as the input of the sideslip angle estimation in next section. The four-wheel independent drive electric vehicle is actuated by four in-wheel motors, and each in-wheel motor can be supervised separately. The single EDWM is not only an independent control unit, but also an independent information unit. We can make full use of the electric-drive characteristics of four-wheel independent drive electric vehicle and use the current sensor, revolution speed sensor, and voltage sensor to estimate the longitudinal force, which helps to reduce the cost of virtual longitudinal force sensor.

As we can see, in formula (11), the longitudinal force is the unknown input of EDWM, and the measurements of current sensor and revolution speed sensor are easy to be disturbed by noise. Therefore, before estimating the longitudinal forces, we need to design model-state observer. A sliding mode observer is designed to achieve the accurate estimation of model states

$$
\begin{aligned}
& \dot{\hat{x}}_{f}=A_{f} \widehat{x}_{f}+B_{f} u_{f}+H\left(y_{f}-\widehat{y}_{f}\right)+\lambda \\
& \hat{y}_{f}=C_{f} \widehat{x}_{f}
\end{aligned}
$$

where matrix $H$ is the observer gain and $\lambda$ is the switching term of sliding mode observer. In consideration of the influences of switching term, the chattering phenomena are unavoidable in sliding mode observer. To reduce the influences chattering phenomena and improve the estimation accuracy, the boundary layer of sliding mode is applied to design the switching term

$$
\lambda= \begin{cases}-\mu \frac{e_{y}}{\left\|e_{y}\right\|}, & \left\|e_{y}\right\|>\xi_{1} \\ -\mu \frac{e_{y}}{\xi_{1}}, & \left\|e_{y}\right\| \leq \xi_{1}\end{cases}
$$

where $e_{y}=\widehat{y}_{f}-y_{f}$ represents the measurement error, $\xi_{1}$ represents the boundary layer thickness of sliding mode observer, and $\mu$ represents the gain matrix of the switching term.

In order to prove the convergence of model-state observer, the Lyapunov function is designed as $V=$ (1/ 2) $e_{x}^{T} e_{x}$, where $e_{x}=\widehat{x}_{f}-x_{f}$ represents the model state error. The differential formula of Lyapunov function can be computed as

$$
\begin{aligned}
\dot{V} & =e_{x}^{T}\left(A_{f} \widehat{x}_{f}-A_{f} x_{f}-H C_{f} e_{x}+\lambda-D_{f} d_{f}\right) \\
& =e_{x}^{T}\left(\Delta f-H C_{f} e_{x}+\lambda-D_{f} d_{f}\right) \\
& \leq-\|H\|\left\|C_{f}\right\|\left\|e_{x}\right\|^{2}-\|e\|\left(\|\mu\|-\left\|D_{f} d_{f}\right\|-\|\Delta f\|\right)
\end{aligned}
$$

where $\Delta f=A_{f} \widehat{x}_{f}-A_{f} x_{f}$. It can be found that the smoothness of the estimated results of the designed sliding mode observer is positively related to the width of the boundary layer. The larger the width of the boundary layer is, the less chattering is. But, the boundary layer will cause the sliding mode deviating from the steady state at the same time. As one can see in formula (15), to promote the existence of sliding mode and the asymptotic stability of sliding mode observer simultaneously, the gain matrix $\mu$ is designed to make the condition $\|\mu\|>\left\|D_{f} d_{f}\right\|+\|\Delta f\|$ satisfied and the inequality $\dot{V}_{1}<0$ hold.

The observers with good performance are required to deal with unpredictable interference factors. The actual driving conditions of vehicles are complex, and there are always some unavoidable disturbances and environmental factors that will weaken the estimation performance of the designed observer. Accordingly, the maximum value of $\left\|D_{f} d_{f}\right\|$ is almost unpredictable and varies with time. With that in mind, in order to achieve appropriate $\mu$ to reduce the estimation error, for the sake of conservatism, the value of $\mu$ should be as large as possible. But oversized $\mu$ is very likely to cause unnecessary chattering in the estimation results. To alleviate this contradiction, a compromising and adaptive way must be chosen. An iterative algorithm for switching gain matrix is proposed, which adjusts the switching gain matrix in real time according to the change of model unknown input, and the switching gain matrix is designed as

$$
\begin{aligned}
\mu_{\mathrm{i}, k+1} & (t) \\
= & \mu_{\mathrm{i}, k}(t)+K_{i}|| e_{y i, k}(t)\left|-\xi_{0}\right| \\
& \quad \times \operatorname{sgn}\left\{\left(\left|e_{y i, k}(t)\right|-\xi_{0}\right) \times\left(\left|e_{y i, k-1}(t)\right|-\xi_{0}\right)\right\}
\end{aligned}
$$


where $i=1,2,3,0<\left\|\xi_{0}\right\| \leq \xi_{1}$ and $K_{i}$ represents the gain of $\mu_{i}$ and can be applied to adjust the convergence speed of switching gain. By analyzing formula (16), one can see that if the observer goes beyond the sliding mode boundary layer, the calculation result of sign function is 1 , and the gain of switching term will increase. If the observer is within the sliding mode boundary layer, the calculation result of sign function is -1 , and the gain of switching term will decrease.

As mentioned earlier, the longitudinal force is the unknown input of EDWM. By observing the EDWM, we can see that the lower part of matrix $B_{f}$ and the upper part of matrix $D_{f}$ are zero. Therefore, in order to facilitate the design of longitudinal force observer, the EDWM in formula (11) is decoupled and the subsystem model can be expressed as

$$
\begin{aligned}
& \dot{x}_{1 f}=A_{1 f} x_{f}+B_{1 f} u_{f} \\
& \dot{x}_{2 f}=A_{2 f} x_{f}+D_{2 f} d_{f}
\end{aligned}
$$

where formula (17) is longitudinal-force-free. According to formula (17) and formula (18), the analytic formula of longitudinal force is deduced as

$$
d_{f}=D_{2 f}^{-1}\left(\dot{x}_{2 f}-A_{2 f} x_{f}\right)=D_{2 f}^{-1}\left(\dot{\bar{x}}_{2 f}-A_{2 f} \widehat{x}_{f}\right)
$$

where $\widehat{x}_{f}$ has been calculated by the sliding mode observer in (13).

With the system states of EDWM being obtained by the observer in formula (13), the longitudinal force estimation method can be developed, and then the longitudinal force estimation results can be used to as the inputs of sideslip angle estimator in further detail below. The idea of timedelay estimation method is applied to longitudinal force estimation. A small enough time step is defined as $T$ and assumed as the iteration cycle of longitudinal force estimation system. Thus, the longitudinal force estimation method can be presented as

$$
\begin{aligned}
\widehat{d}_{f}(t) & =D_{2 f}^{-1}\left(\dot{\hat{x}}_{2 f}(t)-A_{2 f} \widehat{x}_{f}(t)\right) \\
& =D_{2 f}^{-1}\left(\frac{\widehat{x}_{2 f}(t)-\widehat{x}_{2 f}(t-T)}{T}-A_{2 f} \widehat{x}_{f}(t)\right) \\
& =-\frac{J}{r}\left(\frac{\widehat{x}_{2 f}(t)-\widehat{x}_{2 f}(t-T)}{T}-A_{2 f} \widehat{x}_{f}(t)\right)
\end{aligned}
$$

3.2. Vehicle Sideslip Angle Estimation Based on Adaptive Kalman Filter Algorithm. According to formulas (1), (2), and (3), the 3-DOF vehicle dynamic model can be expressed as the following nonlinear discrete state space equation:

$$
\begin{aligned}
x_{k+1} & =A_{k} x_{k}+B_{k} u_{k}+w_{k} \\
y_{k} & =C_{k} x_{k}+v_{k}
\end{aligned}
$$

where $x_{k+1}$ is the discrete system state vector and $y_{k+1}$ represents the discrete system output vector. $A_{k+1}, B_{k+1}$, $C_{k+1}$ represent the state transition matrix, input matrix, and measurement matrix, respectively. $w_{k}$ and $v_{k}$ represent the uncorrelated white noise vectors of zero mean, $w_{k}$ is the system noise with the covariance matrix of $Q_{k}$, and $v_{k}$ is the measurement noise with the covariance matrix of $R_{k}$.

The extended Kalman filter (EKF) is very easy to see in the literatures of vehicle driving state estimation and has been proved to be effective in practical applications. According to state space equation in formula (21), the recursive steps of EKF can be written as follows.

Step 1 (state information estimation). First, the predictive value under a fixed step length is calculated:

$$
\widehat{x}_{k+1 / k}=A_{k} \widehat{x}_{k}+B_{k} u_{k}
$$

Then, the prediction error variance with the step length is obtained:

$$
P_{k+1 / k}=\Phi_{k} P_{k / k} \Phi_{k}^{T}+Q_{k}
$$

where $P_{k}$ is the covariance matrix of system state error, $Q_{k}$ is the covariance matrix of process noise, $\Phi_{k}=I+A_{k} T, T$ represents the iteration cycle of adaptive Kalman filter, and is equal to the iteration cycle of LFO.

Step 2 (a priori estimation correction). First, the feedback gain matrix of state estimation is further calculated based on the previously predicted variance.

$$
K_{k+1}=P_{k+1 / k} C_{k+1}^{T}\left(C_{k+1} P_{k+1 / k} C_{k+1}^{T}+R_{k+1}\right)^{-1}
$$

where $K_{k+1}$ is the feedback gain matrix. Then, according to the feedback gain matrix, the state filter estimation value under the current time is calculated.

$$
\widehat{x}_{k+1 / k+1}=\widehat{x}_{k+1 / k}+K_{k+1}\left[y_{k+1}-C_{k+1} x_{k+1 / k}\right]
$$

Finally, the variance of the filtering estimation error at the next moment is calculated again, and the next iteration estimation is carried out.

$$
P_{k+1 / k+1}=\left(I-K_{k+1} C_{k+1}\right) P_{k+1 / k}
$$

However, the actual driving conditions of vehicles are relatively complicated and the vehicle estimation results are easily influenced by environmental factors. The EKF has the disadvantages of large error and slow convergence under these circumstances. In order to solve this problem, the AAKF is proposed to estimate the vehicle running state. The algorithm uses time-varying attenuation factor to weaken the influence of past data to the current filter state, so as to improve the robustness and accuracy of the filter. Moreover, the algorithm can adjust the covariance of process noise and measurement noise adaptively, which helps to improve the accuracy and convergence speed of the filter. The data flow chart of AAKF is shown in Figure 3, and the recursive steps of AAKF can be written as follows.

Step 1 (initial conditions).

$$
\begin{aligned}
& x_{0 / 0}=E\left(x_{0}\right), \\
& P_{0 / 0}=E\left[\left(x-x_{0}\right)\left(x-x_{0}\right)^{T}\right]
\end{aligned}
$$




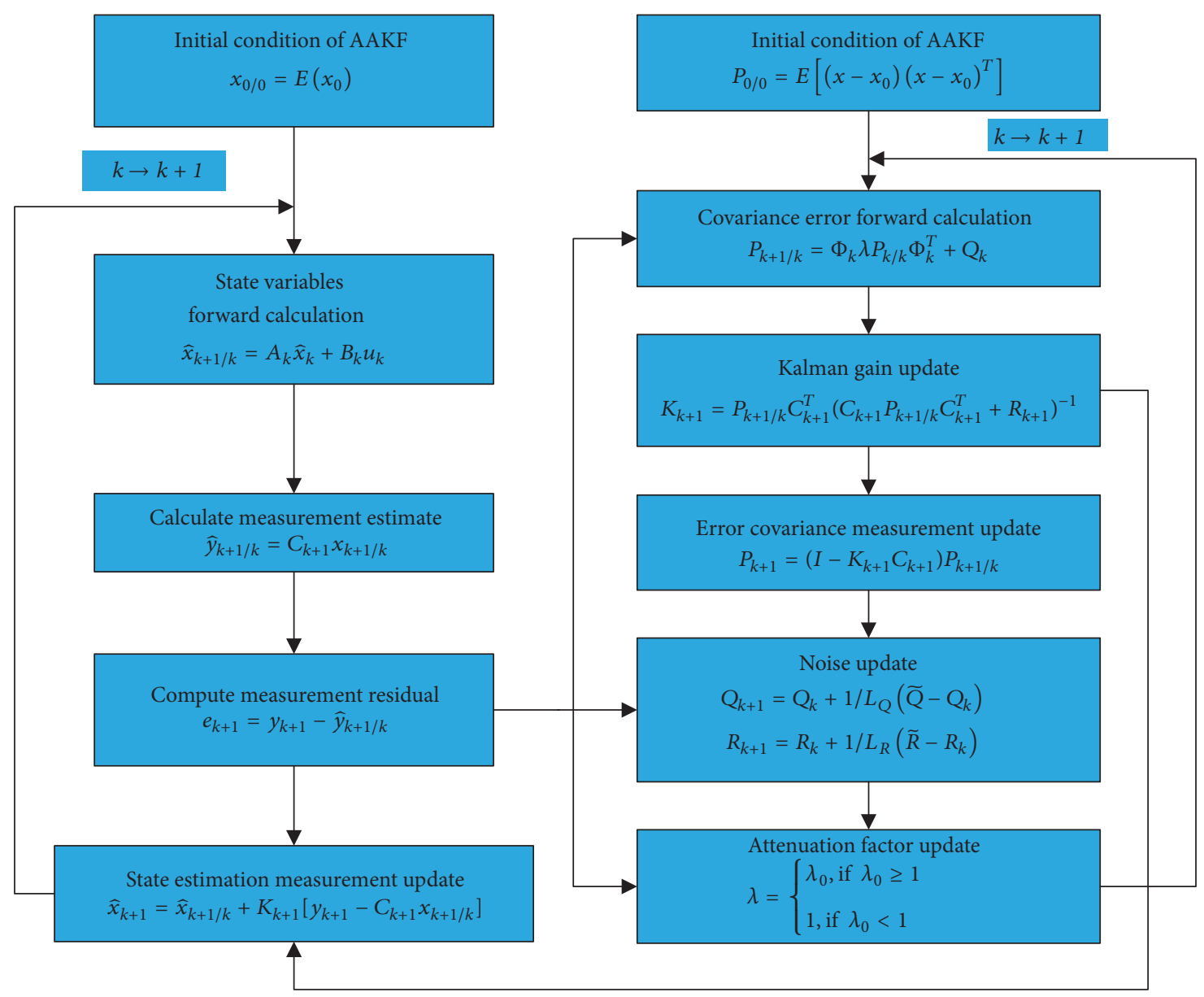

FIGURE 3: Data flow chart of AAKF.

Step 2 (forward calculations of state variables and error covariance).

$$
\begin{aligned}
& \widehat{x}_{k+1 / k}=A_{k} \widehat{x}_{k}+B_{k} u_{k} \\
& P_{k+1 / k}=\Phi_{k} \lambda P_{k / k} \Phi_{k}^{T}+Q_{k}
\end{aligned}
$$

where $\lambda$ is the attenuation factor. In Step 2, in order to improve the robustness and accuracy of filter, the attenuation factor is applied. When the state estimation measurement is updated, the Kalman gain is adjusted by the attenuation factor to reduce the weight of the past data, so as to realize the function of weakening the influence of the past data on the current filter value.

Step 3 (update of Kalman gain).

$$
K_{k+1}=P_{k+1 / k} C_{k+1}^{T}\left(C_{k+1} P_{k+1 / k} C_{k+1}^{T}+R_{k+1}\right)^{-1}
$$

Step 4 (measurement updates of state estimation and error covariance).

$$
\begin{aligned}
& \hat{x}_{k+1}=\widehat{x}_{k+1 / k}+K_{k+1}\left[y_{k+1}-C_{k+1} x_{k+1 / k}\right] \\
& P_{k+1}=\left(I-K_{k+1} C_{k+1}\right) P_{k+1 / k}
\end{aligned}
$$

Step 5 (updates of measurement noise and system noise).

$$
\begin{aligned}
Q_{k+1} & =Q_{k}+\frac{1}{L_{Q}\left(\widetilde{Q}-Q_{k}\right)} \\
R_{k+1} & =R_{k}+\frac{1}{L_{R}\left(\widetilde{R}-R_{k}\right)}
\end{aligned}
$$

where $\widetilde{Q}=K_{k+1}\left(y_{k+1}-C_{k+1} x_{k+1 / k}\right)\left(y_{k+1}-C_{k+1} x_{k+1 / k}\right)^{T} K_{k+1}^{T}-$ $P_{k+1}+A_{k+1} P_{k} A_{k+1}^{T}, \widetilde{R}=\left(y_{k+1}-C_{k+1} x_{k+1 / k}\right)\left(y_{k+1}-\right.$ $\left.C_{k+1} x_{k+1 / k}\right)^{T}-C_{k+1} P_{k} C_{k+1}^{T}$ and $L_{Q}$ and $L_{R}$ represent the adjustment window value of system noise and measurement noise, respectively. Therefore, the time-varying attenuation factor is used to suppress the memory length of filter to make full use of the current observation data and reduce the influence of old measurements. When $\lambda_{0} \leq 1$, the adaptive Kalman filter is equivalent to the conventional Kalman filter. When $\lambda_{0}>1$, the error covariance matrix with adaptive fading factor is larger than that without fading factor. One can see that, by adaptively adjusting the covariance of process noise and measurement noise, the filter can better adapt to the change of noise statistical characteristics and improve the accuracy, convergence speed, and adaptability of the filter. 
Step 6 (updates of attenuation factor).

$$
\lambda= \begin{cases}\lambda_{0}, & \text { if } \lambda_{0} \geq 1 \\ 1, & \text { if } \lambda_{0}<1\end{cases}
$$

where $\lambda_{0}=\operatorname{tr} N_{k} / \operatorname{tr} M_{k}, N_{k}=E\left(\left(y_{k}-C_{k} x_{k / k-1}\right)\left(y_{k}-\right.\right.$ $\left.\left.C_{k} x_{k / k-1}\right)^{T}\right)-C_{k} B_{k} Q_{k} B_{k}^{T} C_{k}^{T}-R_{k}, M_{k}=C_{k} A_{k} P_{k} A_{k}^{T} C_{k}^{T}$. When the model is accurate, the output residual sequence of Kalman filter is not self-correlated Gauss white noise sequence, so it is required that the residual sequence at different times be orthogonal to each other. As we can see in formula (35), the adaptive adjustment of attenuation factor can be realized.

In the design process of AAKF, the input variables of the AAKF can be expressed as

$$
u=\left[\begin{array}{lllllllll}
\delta & F_{x 1} & F_{x 2} & F_{x 3} & F_{x 4} & F_{y 1} & F_{y 2} & F_{y 3} & F_{y 4}
\end{array}\right]^{T}
$$

The system state of AAKF is

$$
x=\left[\begin{array}{lll}
v_{x} & v_{y} & \gamma
\end{array}\right]^{T}
$$

And the measurement output of AAKF is

$$
y=\left[\begin{array}{ll}
a_{x} & a_{y}
\end{array}\right]^{T}
$$

where $a_{x}$ and $a_{y}$ represent the longitudinal acceleration and lateral acceleration of vehicle, respectively, and satisfy the following formulas:

$$
\begin{aligned}
& a_{x}=\dot{v}_{x}-\gamma v_{y} \\
& a_{y}=\dot{v}_{y}+\gamma v_{x}
\end{aligned}
$$

The global vehicle driving state estimation strategy is shown in Figure 4. As we can see in Figure 4, four longitudinal force observers are designed for four electric driving wheels of vehicle, and the previously designed longitudinal force observers can be regarded as the VLFSs at this time. The measurements of current, wheel speed, and voltage sensors are used as the inputs of VLFSs to compute the longitudinal forces. The steering angle of front wheel, longitudinal and lateral acceleration of vehicle measured by vehicular sensors, the estimated longitudinal tire forces, and the lateral tire force obtained by tire model are used as the input of designed AAKF to estimate the longitudinal speed, lateral speed, and yaw rate of vehicle. And then, the vehicle sideslip angle estimation can be achieved by following formula.

$$
\beta=\arctan \left(\frac{v_{y}}{v_{x}}\right)
$$

\section{Simulation Results}

In this section, to assess the effectiveness of the proposed vehicle running state estimation method in this work, the simulations are carried out on a high-fidelity CarSimSimulink joint simulation platform. The CarSim is professional simulation software specially for vehicle dynamics and is used to offer the whole vehicle dynamic model in simulation, and the estimations of longitudinal force, longitudinal vehicle speed, lateral vehicle speed, yaw rate, and sideslip angle are achieved in Matlab/Simulink software. The CarSimSimulink joint simulation model is shown in Figure 5. The parameters of vehicle model, tire model, and in-wheel motors model are listed in Table 1. In order to demonstrate the superiority of the proposed estimation method in this paper, in the verification process of longitudinal force estimation method, a common-used unknown input observer is applied to compare with the designed VLFS. In addition, the EKF is used to estimate vehicle running states and compare with the designed AAKF.

In case study 1 , the Double Lane Changes (DLC) manoeuvre, as shown in Figure 6, is actualized to verify the proposed estimation method. In this simulation, the road friction coefficient is set to be 0.6 , and the vehicle speed maintains at a constant of $20 \mathrm{~m} / \mathrm{s}$. The comparison of estimated longitudinal force and actual longitudinal force is shown in Figure 7, in which the estimation results of proposed longitudinal force observer, the estimation results of common unknown input observer, and the actual longitudinal force in CarSim are denoted as "VLFS," "UIO," and "CarSim," respectively. In Figure 7, it can be seen that the designed VLFS can precisely estimate the longitudinal force in real-time against the changes in actual driving conditions. However, the estimation accuracy and dynamic tracking capability of UIO is lower than VLFS. From Figures $7(\mathrm{a})-7(\mathrm{~d})$, the partial enlarged drawings of $1 s-3 s, 3 s-5 s, 5 s-7 s$, and $7 s-9 s$ are displayed, in which we can see that the estimation errors of UIO are larger and the estimation results of UIO in Figures 7(b)-7(d) have a certain degree of time lag. The estimated results of longitudinal vehicle speed, lateral vehicle speed, yaw rate, and vehicle sideslip angle are shown in Figure 8. On the whole, the estimation results of EKF and AAKF are satisfactory with relatively high estimation accuracy. From the partial enlarged drawing we can see that the estimation effect of AAKF is obviously better than that of EKF. For example, in Figure 8(a), the estimated longitudinal vehicle speed of AAKF has smaller fluctuation compared with that of EKF. And according to Figures 8 (b), 8(c), and 8(d), one can see that, at the vertex of the curve fluctuation, the deviation of EKF estimation results is relatively serious, which indicates that the AAKF method has better real-time tracking ability and estimation accuracy when the vehicle driving state changes sharply or the vehicle driving conditions are complex.

In order to quantitatively reflect the estimation performance of the proposed estimation method, the peak of relative error (PRE) and the error of root mean square (ERMS) between actual value and estimated value are used for comparative evaluation and can be computed by the following formulas:

$$
\begin{aligned}
\operatorname{PRE}(x) & =x_{k / p}-x_{\mathrm{CarSim} / p} \\
\operatorname{ERMS}(x) & =\sqrt{\frac{1}{N} \sum_{k=1}^{N}\left(x_{k}-x_{\mathrm{CarSim}}\right)^{2}}
\end{aligned}
$$


TABLE 1: Parameters of vehicle model, tire model, and in-wheel motors.

\begin{tabular}{lcc}
\hline Symbol & Parameters & Value and units \\
\hline$m$ & Vehicle mass & $710 \mathrm{~kg}$ \\
$r$ & Effective radius of wheel & $0.245 \mathrm{~m}$ \\
$l_{f}$ & Distances from vehicle gravity center to the front axle & $0.795 \mathrm{~m}$ \\
$l_{r}$ & Distances from vehicle gravity center to the rear axle & $0.975 \mathrm{~m}$ \\
$b_{f}, b_{r}$ & Half treads of the front $(\mathrm{rear})$ wheels & $0.775 \mathrm{~m}$ \\
$C_{f}$ & Equivalent cornering stiffness of front wheel & $60000 \mathrm{~N} / \mathrm{rad}$ \\
$C_{r}$ & Equivalent cornering stiffness of rear wheel & $40000 \mathrm{~N} / \mathrm{rad}$ \\
$I_{z}$ & Moment of inertia & $1000 \mathrm{~kg} \cdot \mathrm{m}^{2}$ \\
$R$ & Equivalent resistance of winding & $0.688 \Omega$ \\
$K_{a}$ & Inverse electromotive force coefficient & $0.06 \mathrm{Nm} / \mathrm{A}$ \\
$K_{t}$ & Motor torque constant & $11.43 \mathrm{Nm} / \mathrm{A}$ \\
$J$ & Sum of inertia moment of wheel and motor & $7.143 \mathrm{~kg} \cdot \mathrm{m}^{2}$ \\
$b$ & Damping coefficient & $0.643 \mathrm{Nm} \cdot \mathrm{sec} / \mathrm{rad}$ \\
$L$ & Equivalent inductance of winding & $0.125 \mathrm{H}$ \\
\hline
\end{tabular}

where $N$ is the number of samples, $x_{k}$ is the estimated vehicle running states of UIO, VLFS, EKF, and AAKF at the $k$ th sample, $x_{\text {CarSim }}$ represents the actual vehicle running states obtained by CarSim software at the $k$ th sample, $x_{k / p}$ is the peak value of $x_{k}$, and $x_{\mathrm{CarSim} / \mathrm{p}}$ is the peak value of $x_{\mathrm{CarSim}}$. The comparison of PRE between UIO and VLFS and the comparison of PRE between EKF and AAKF are shown in Table 2. The comparison of ERMS between UIO and VLFS and the comparison of PRE between EKF and AAKF are shown in Table 3. According to Table 2, it can be found that the PRE of UIO is about three times as large as the PRE of VLFS, which means that the maximum estimation error of VLFS is much less than the maximum estimation error of UIO, and the estimation accuracy of VLFS is quite acceptable. In addition, the PRE of AAKF is also obviously less that of EKF, and the accuracy of AAKF is also proved. In Table 3, one can see that the ERMS of VLFS is less than that of UIO, and the ERMS of AAKF is less than that of EKF, which indicates that the designed estimation method has better overall estimation effect, the estimation error of VLFS and AAKF fluctuates less, and the estimation performance of VLFS and AAKF is more stable as a whole.

In case study 2, the fishhook simulation manoeuvre is implemented for further verification in intense driving conditions. In fishhook manoeuvre, the vehicle speed is $15 \mathrm{~m} / \mathrm{s}$, the road friction coefficient is set to be 1.0 , and the steering wheel angle is shown in Figure 9. The longitudinal force estimation results are shown in Figure 10. It can be found that the chattering phenomena in VLFS are alleviated compared with UIO when the steering wheel angle is large during $3 \mathrm{~s}$ to $5 \mathrm{~s}$. When the steering wheel angle is constant at $-90 \mathrm{deg}$, the estimation results of VLFS is more accurate than UIO. The vehicle running state estimation results in fishhook manoeuvre are shown in Figure 11. As shown in Figure 11, the same as case study 2, the estimation performance of AAKF is also satisfactory and better than UIO. In Table 4, we can see that the PRE of UIO is greater than 10 and the PRE of VLFS is about 3. Furthermore, the PRE of EKF is also larger than AAKF. In Table 5, the ERMS of UIO and EKF are also larger than VLFS and AAKF, which proves that the proposed estimation method can still maintain reliable estimation performance under more complex driving condition.

\section{Experimental Results}

The experimental test is implemented for further validation about the designed observer in this section. The autonomous four-wheel independent drive electric vehicle used in experiment was converted from a centralized drive vehicle. The modified experimental vehicle is actuated by four in-wheel motors and the rated power, maximum torque, and maximum speed of each in-wheel motor is $3 \mathrm{KW}, 150 \mathrm{Nm}$, and $750 \mathrm{r} / \mathrm{min}$, respectively. The vehicle power source is a lithium iron phosphate battery pack with $72 \mathrm{~V}$ voltage output, and the vehicle control system is powered by $12 \mathrm{~V}$ power supply transformed by DC/DC converter. A rapid prototyping platform (RPP) is chosen as vehicle control unit, which is used for the model-based control system development. The control system and sensor network of experimental vehicle are shown in Figure 12. Using the CAN bus of RPP, all control signals and sensor signals are recorded by CAN tools of Vehicle SPY3. The front wheel steering angle was transformed from the measured hand steering wheel angle. The vehicle running states were measured by high-precision difference GPS and inertial measurement unit.

Owning to the fact that the sensor for longitudinal force measurement is still unavailable on our experimental vehicle, the verification of VLFS was executed by the experimental data of chassis dynamometer bench test. The logical block diagram of bench test and the scene of vehicle test on chassis dynamometer bench are shown in Figure 13. The principle of chassis dynamometer is to simulate the road virtually via drum. All kinds of resistance encountered by automobiles in normal uniform speed are simulated by loading device. When the vehicle accelerates and glides, the resistance is simulated by the moment of inertia of the flywheel set. The torque and power of the chassis dynamometer and the longitudinal force of the vehicle are measured by a force 


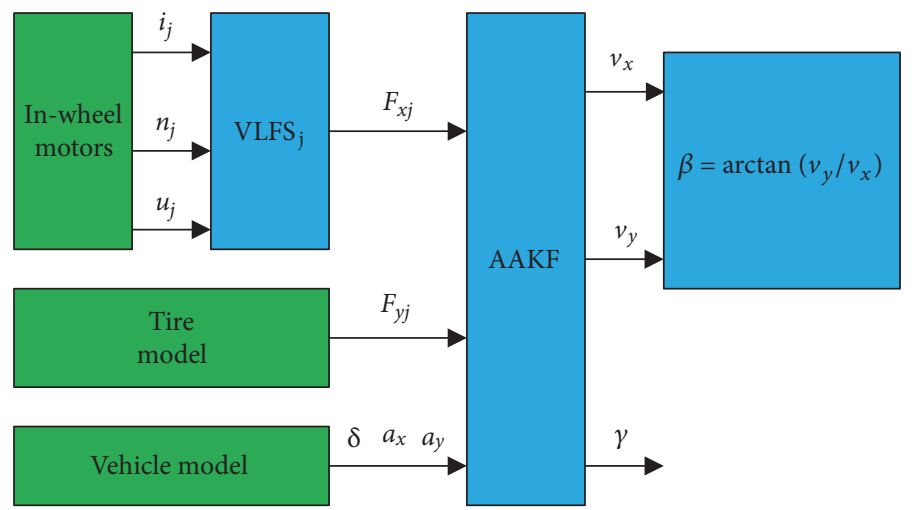

FIgURE 4: Global vehicle driving state estimation strategy.

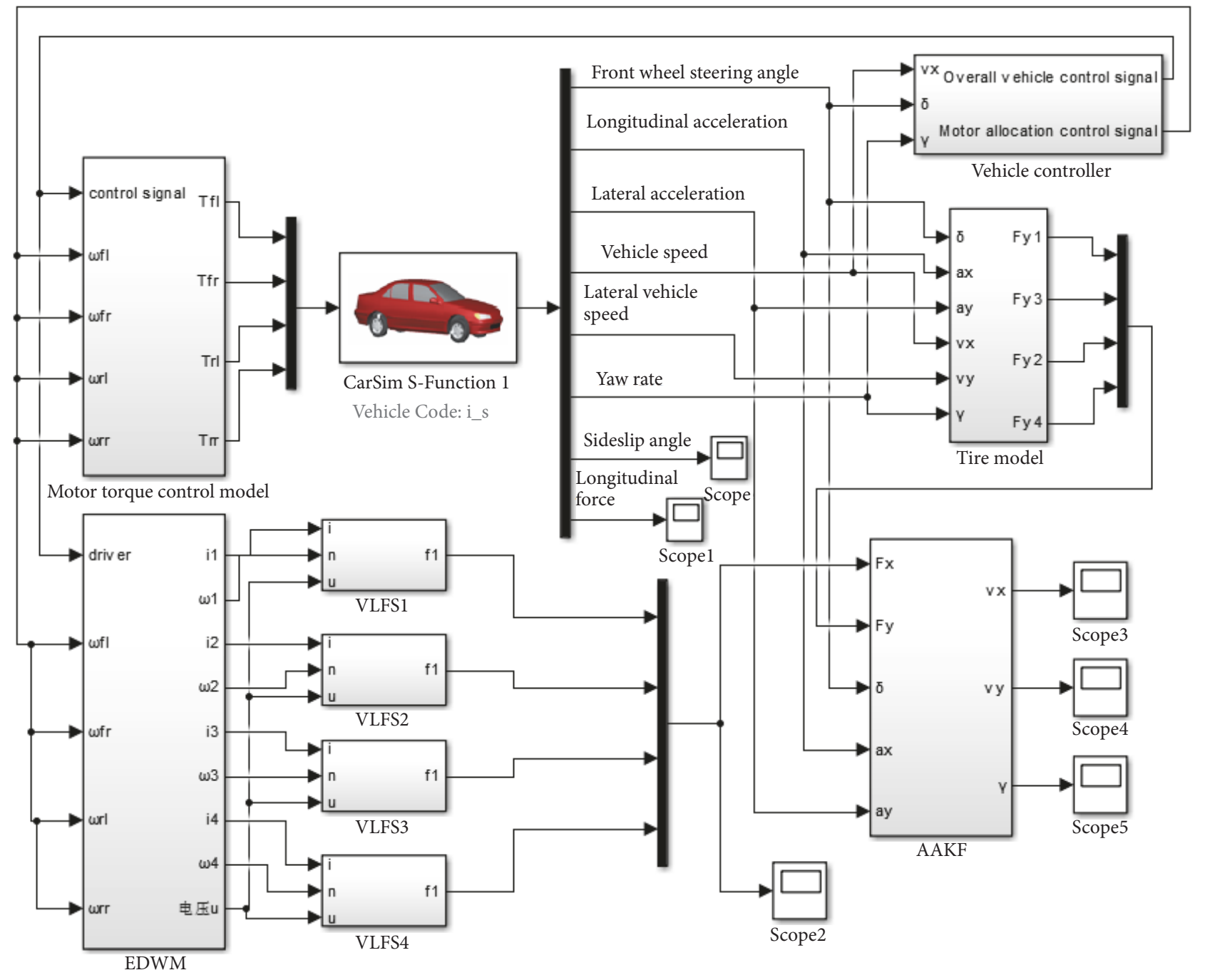

FIGURE 5: Simulation model. 


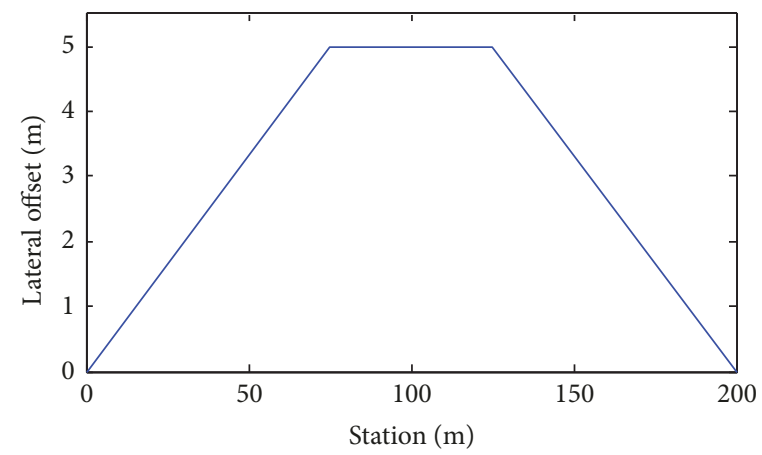

(a)

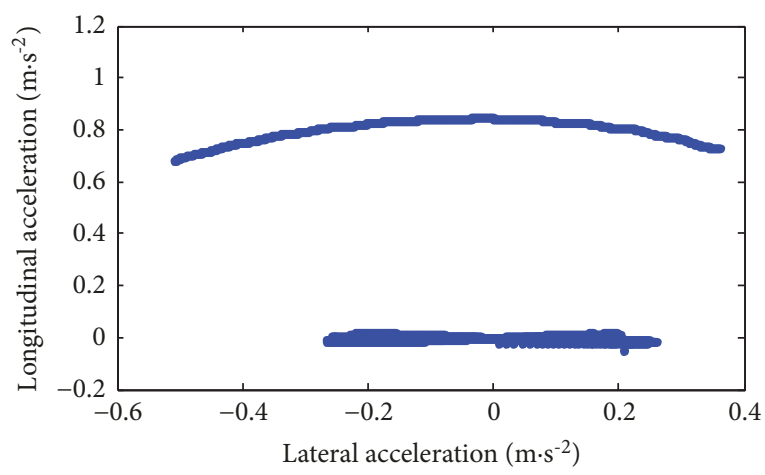

(b)

FIGURE 6: Simulation condition in DLC manoeuvre. (a) Planned trajectory, (b) G-G diagram.
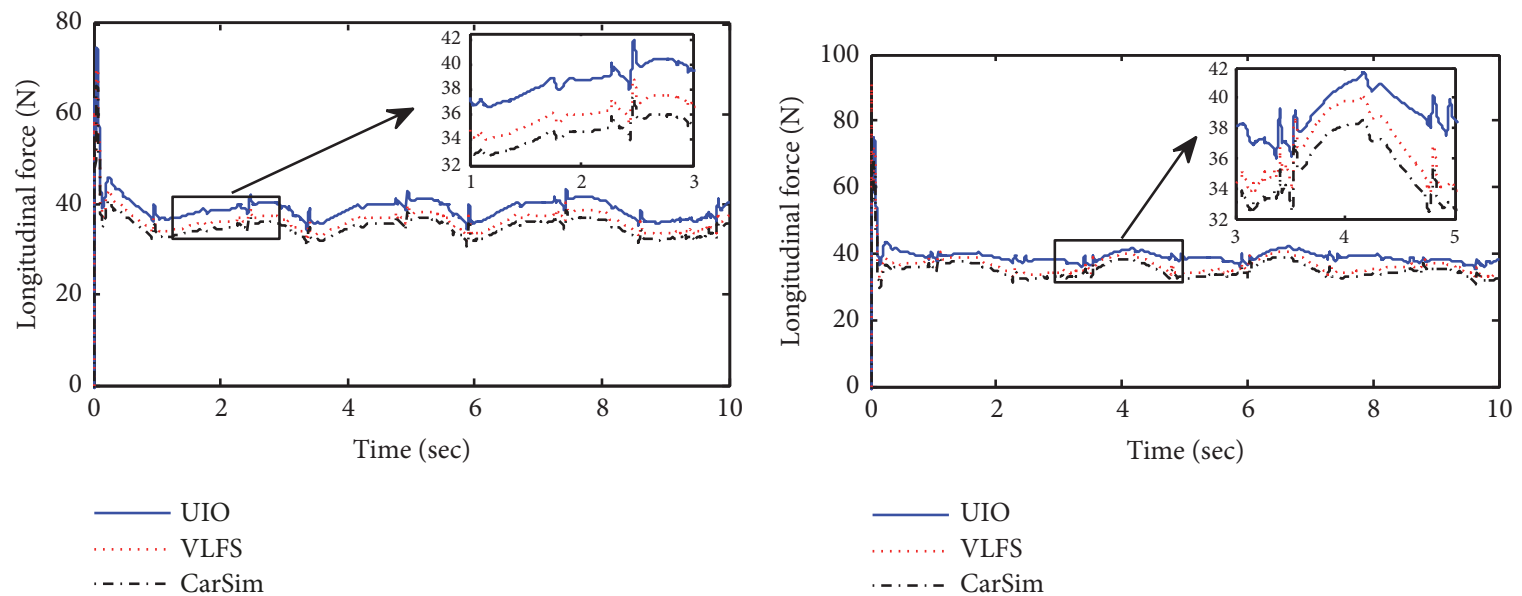

(a)

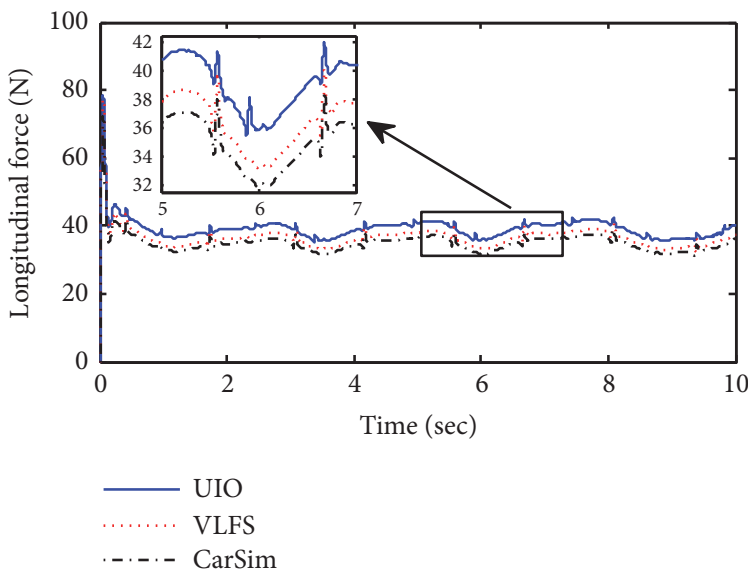

(c)

(b)

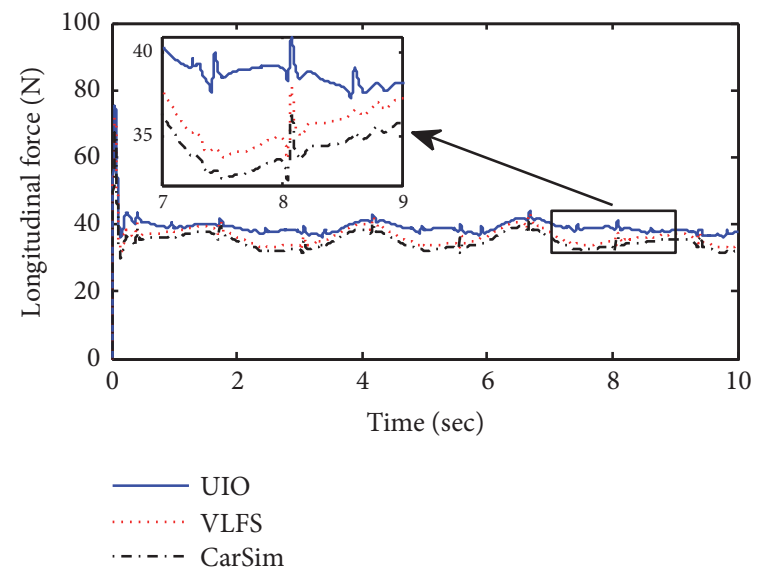

(d)

FIGURE 7: Estimation results of longitudinal force in DLC manoeuvre. (a) Longitudinal force of front-left wheel: $F_{x 1}$, (b) longitudinal force of front-right wheel: $F_{x 2}$, (c) longitudinal force of rear-left wheel: $F_{x 3}$, and (d) longitudinal force of rear-right wheel: $F_{x 4}$.

sensor mounted on a force arm connecting the stator and the dynamometer housing. The measurements of currents, speeds, and voltages of in-wheel motors were obtained by corresponding vehicle-mounted sensors and recorded by the host computer through CAN bus. The longitudinal forces were recorded by the data acquisition system of chassis dynamometer. The experimental data of front-right electric driving wheel is used to verify the designed VLFS in this paper, and the estimation result is shown in Figure 14. It can be found that the proposed VLFS can track the actual longitudinal force in real time, and the estimation accuracy of VLFS is satisfactory. Relatively speaking, the estimation of 


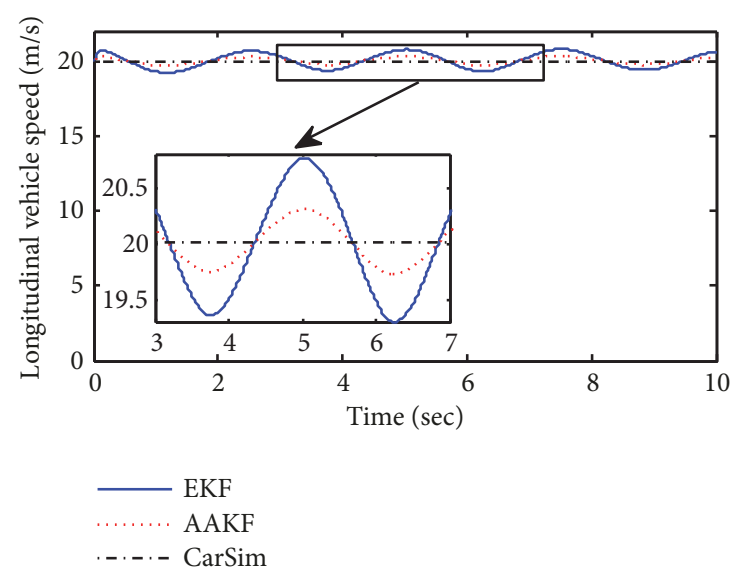

(a)

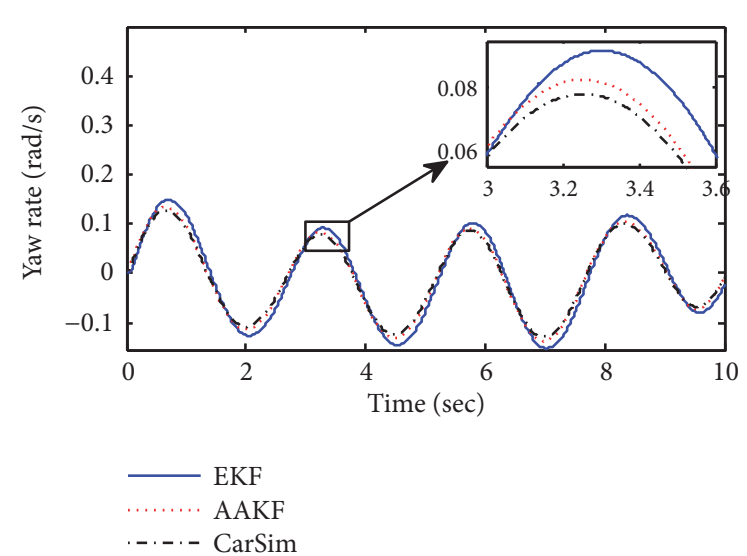

(c)

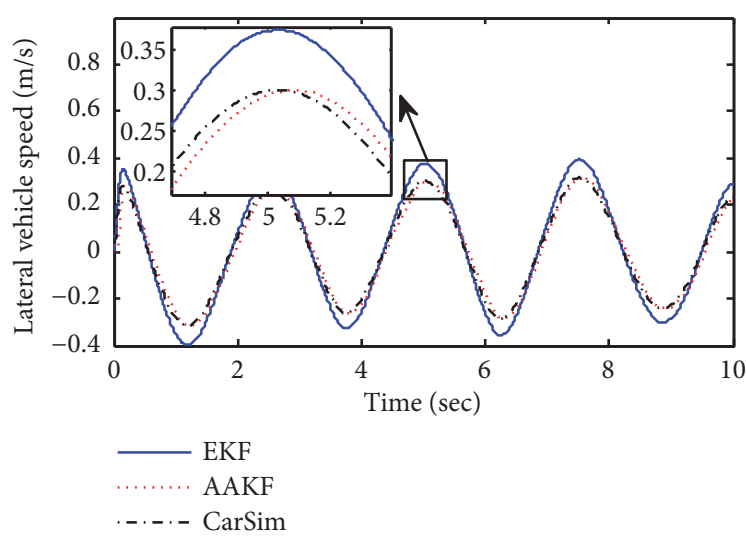

(b)

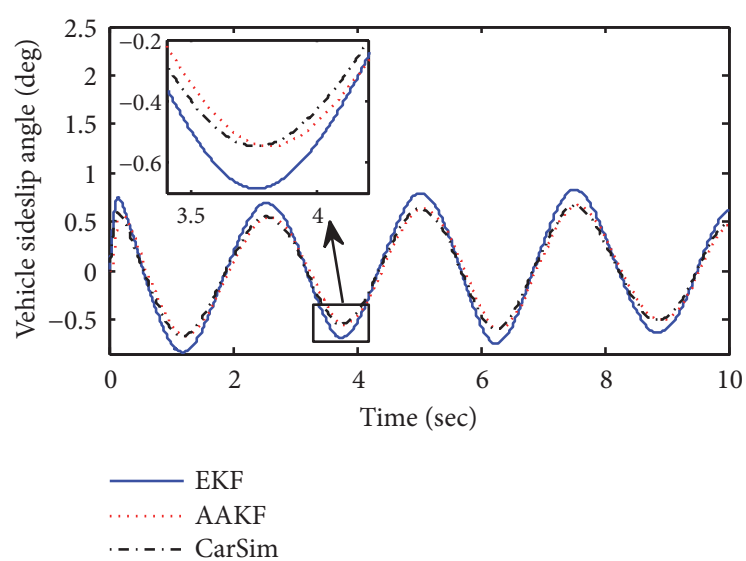

(d)

FIGURE 8: Estimation results of vehicle running states in DLC manoeuvre. (a) Longitudinal vehicle speed, (b) lateral vehicle speed, (c) yaw rate, and (d) vehicle sideslip angle.

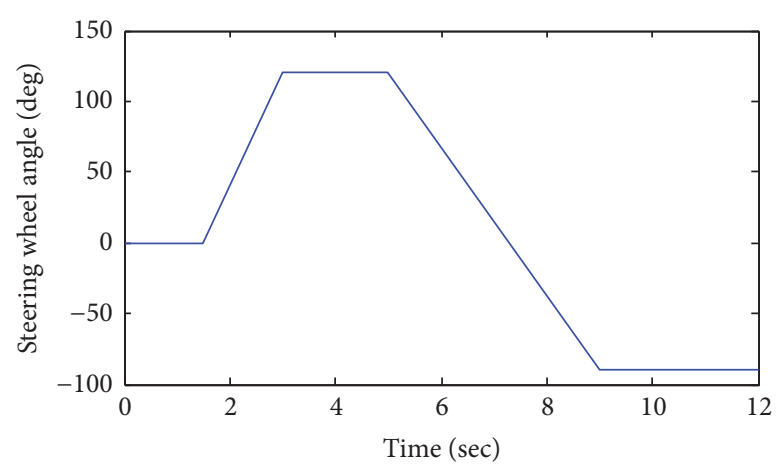

(a)

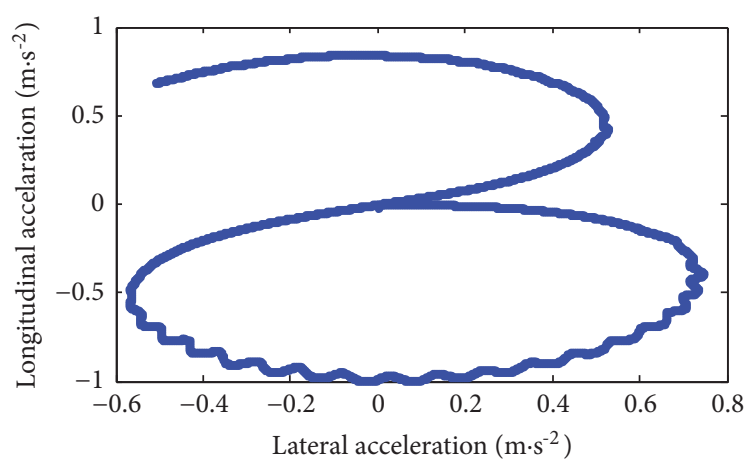

(b)

FIGURE 9: Simulation condition in fishhook manoeuvre. (a) Steering wheel angle, (b) G-G diagram.

UIO has a certain degree of delay, and the fluctuation of UIO is also larger.

Figure 15 shows the experimental vehicle and experimental scene. The experiment was executed in the form of serpentine trajectory, in which 10 traffic piles are placed as the obstacle, and the vehicle speed cruising is regulated by a designed speed controller in RPP. In vehicle driving state estimation verification, the previously validated VLFS is considered as a longitudinal force sensor. Figure 16 expresses the comparison results of vehicle running states in experiment. It can be found that, compared with EKF, the presented AAKF follows the measured longitudinal vehicle speed, lateral vehicle speed, yaw rate, and sideslip angle more accurately and duly. The comparison of PRE and ERMS is shown in Tables 6 and 7, 


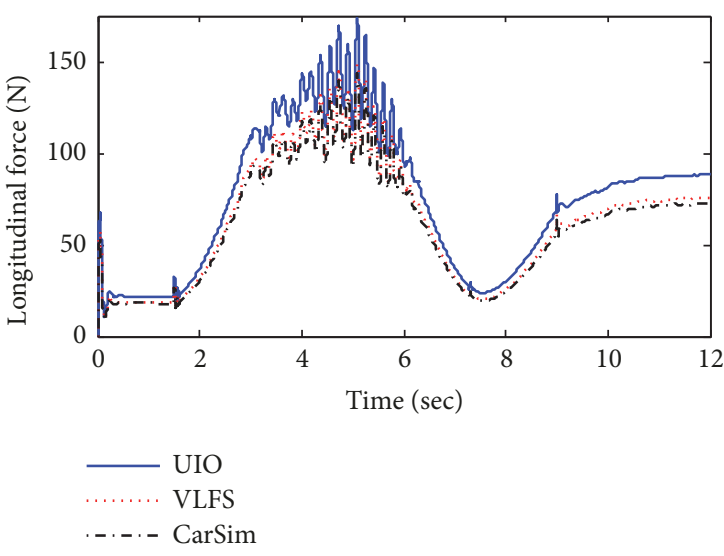

(a)

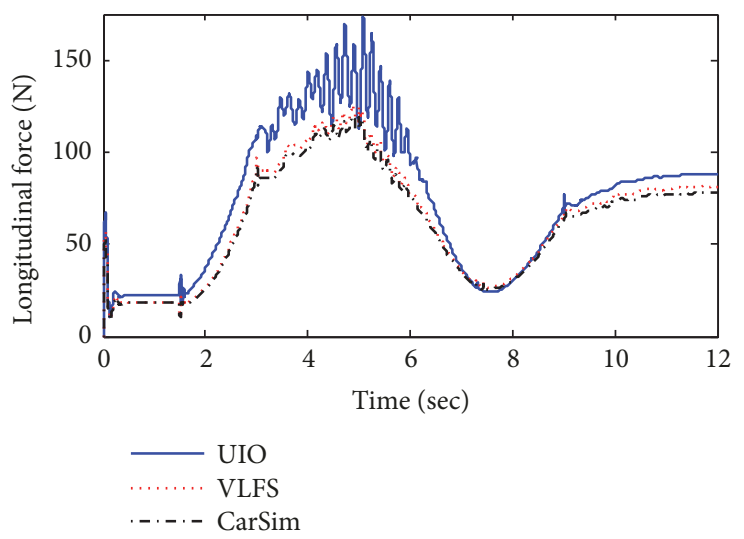

(c)

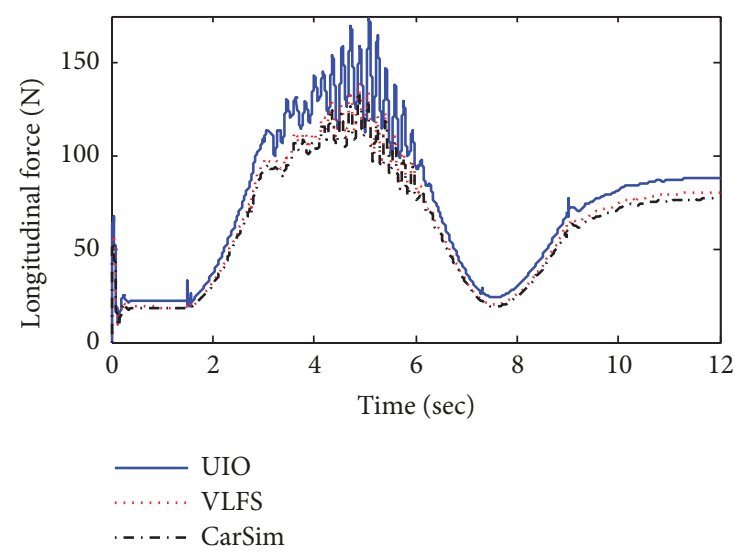

(b)

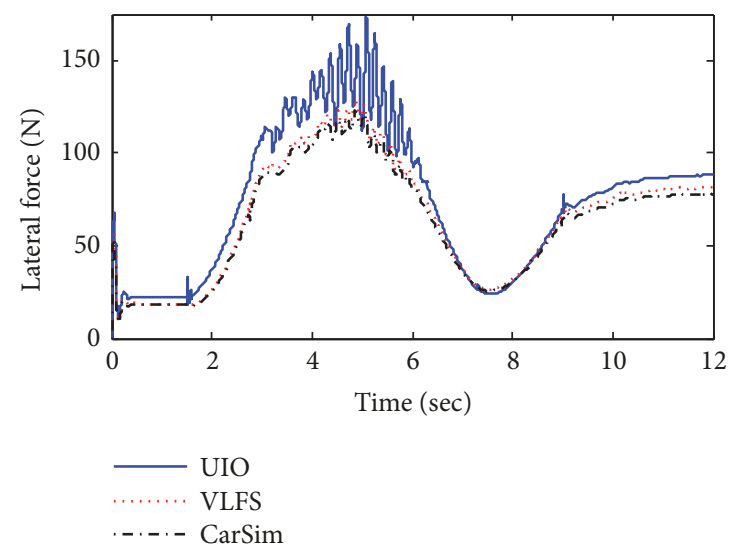

(d)

Figure 10: Estimation results of longitudinal force in fishhook manoeuvre. (a) Longitudinal force of front-left wheel: $F_{x 1}$, (b) longitudinal force of front-right wheel: $F_{x 2}$, (c) longitudinal force of rear-left wheel: $F_{x 3}$, and (d) longitudinal force of rear-right wheel: $F_{x 4}$.

respectively. The same as the simulation results, the PRE and ERMS of UIO or EKF are larger than those of VLFS or AAKF. Notice that the application of VLFS and AAKF guarantees the estimation accuracy and improves the reliability, and the vehicle application effect of VLFS and AAKF in this paper is verified.

\section{Conclusion}

This work presents a novel running states estimation method of autonomous four-wheel independent drive electric vehicle by virtual longitudinal force sensors. The 3-DOF vehicle model, EDWM, and tire model are established. Considering that the EDWM is a nonlinear vehicle model with unknown input, an adaptive high-order sliding mode observer is designed to estimate the state quantity and EDMW, the analytic formula of longitudinal force is obtained by decoupling EDWM, and the longitudinal force estimator is developed by recurrence estimation method. The designed longitudinal force estimator is regarded as a VLFS, and the estimation result is used as the virtual measurement. Then, the AAKF is proposed and applied to vehicle running states estimation.
A high-fidelity CarSim-Simulink joint simulation platform was applied to verify the proposed estimation method and the two case studies like DLC manoeuvre in low adhesion road and fishhook manoeuvre in high adhesion road are actualized. The simulation results show that, compared with UIO and EKF, the proposed VLFS and AAKF can obtain reliable vehicle running state estimation results ensuring higher estimation accuracy and real-time tracking ability. The chassis dynamometer bench test and road test are carried out, and the estimation effect in practical engineering application of proposed estimation method is further verified.

\section{Data Availability}

All readers can access the data by emailing the first author of this article for help. The unavailable data contains the efforts of all authors, and those who use the data need to ask the author's permission.

\section{Conflicts of Interest}

The authors declare that they have no conflicts of interest. 


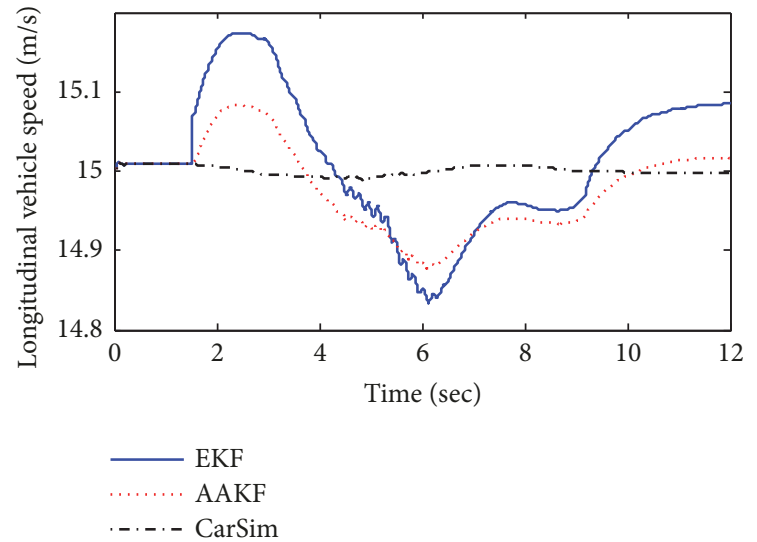

(a)
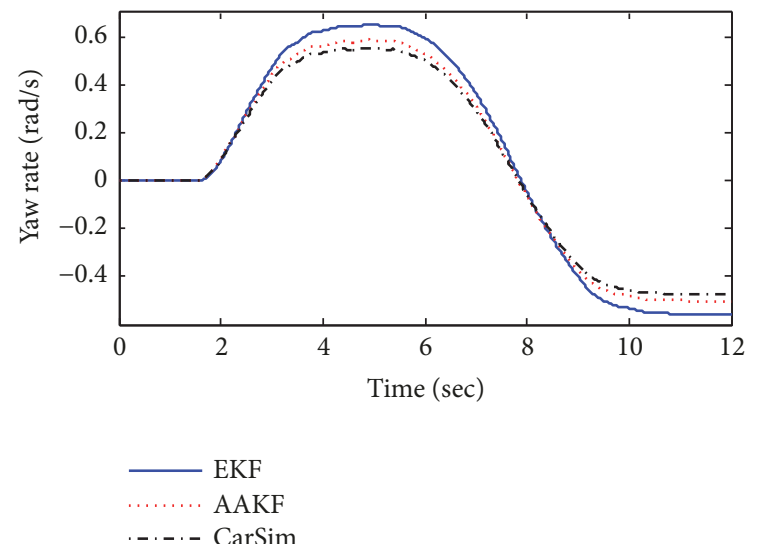

(c)

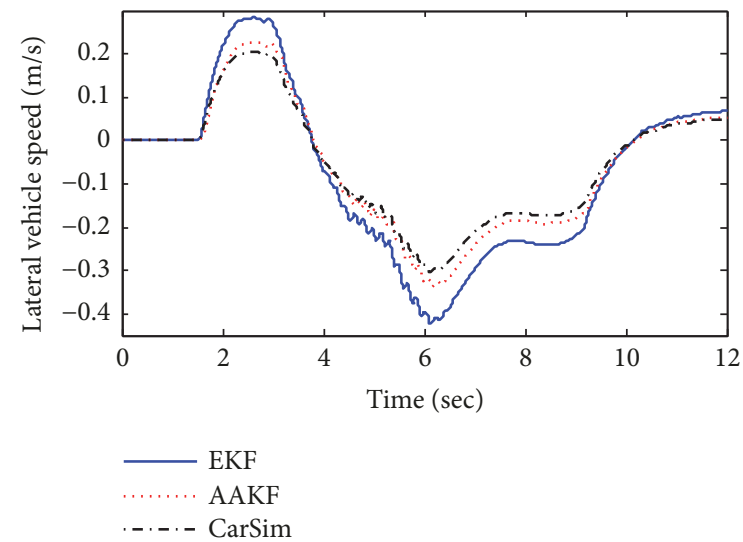

(b)

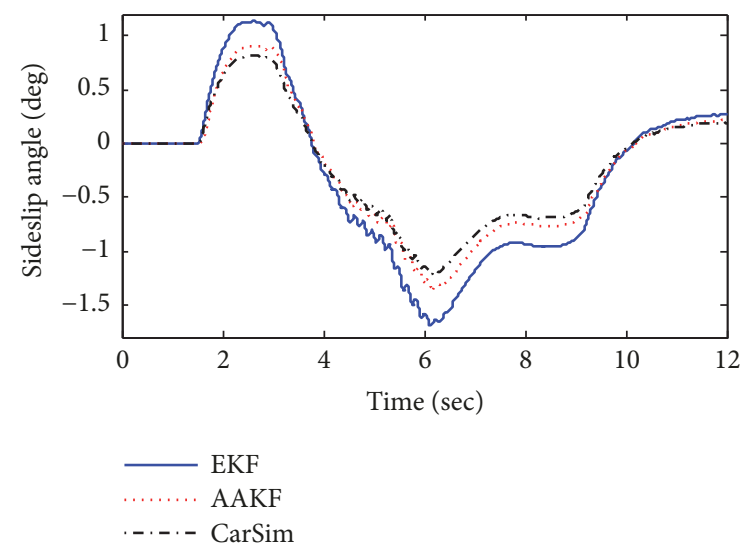

(d)

FIGURE 11: Estimation results of vehicle running states in fishhook manoeuvre. (a) Longitudinal vehicle speed, (b) lateral vehicle speed, (c) yaw rate, and (d) vehicle sideslip angle.

TABLE 2: Comparisons of PRE in DLC manoeuvre.

\begin{tabular}{lcccccccc}
\hline PRE & $F_{x 1}$ & $F_{x 2}$ & $F_{x 3}$ & $F_{x 4}$ & $v_{x}$ & $v_{y}$ & $\gamma$ \\
\hline UIO/EKF & 5.4122 & 5.7783 & 7.2367 & 7.4441 & 0.7304 & 0.0839 & 0.0344 & 0.1345 \\
VLFS/AAKF & 1.7692 & 1.8906 & 2.1909 & 2.0156 & 0.3158 & 0.0213 & 0.0062 & 0.0311 \\
\hline
\end{tabular}

TABLE 3: Comparisons of ERMS in DLC manoeuvre.

\begin{tabular}{|c|c|c|c|c|c|c|c|c|}
\hline ERMS & $F_{x 1}$ & $F_{x 2}$ & $F_{x 3}$ & $F_{x 4}$ & $v_{x}$ & $v_{y}$ & $\gamma$ & $\beta$ \\
\hline UIO/EKF & 0.3907 & 0.3452 & 0.3114 & 0.3355 & 0.2984 & 0.2969 & 0.1831 & 0.2756 \\
\hline VLFS/AAKF & 0.2984 & 0.2656 & 0.2564 & 0.2001 & 0.1602 & 0.1078 & 0.0829 & 0.0993 \\
\hline
\end{tabular}

TABLE 4: Comparisons of PRE in fishhook manoeuvre.

\begin{tabular}{|c|c|c|c|c|c|c|c|c|}
\hline PRE & $F_{x 1}$ & $F_{x 2}$ & $F_{x 3}$ & $F_{x 4}$ & $v_{x}$ & $v_{y}$ & $\gamma$ & $\beta$ \\
\hline UIO/EKF & 15.8028 & 10.3296 & 11.1304 & 10.0974 & 0.1741 & 0.0792 & 0.0984 & 0.3167 \\
\hline VLFS/AAKF & 2.9978 & 3.2393 & 3.1512 & 3.2920 & 0.0829 & 0.0223 & 0.0325 & 0.0926 \\
\hline
\end{tabular}

TABLE 5: Comparisons of ERMS in fishhook manoeuvre.

\begin{tabular}{lcccccccc}
\hline ERMS & $F_{x 1}$ & $F_{x 2}$ & $F_{x 3}$ & $F_{x 4}$ & $v_{x}$ & $v_{y}$ & $\gamma$ \\
\hline UIO/EKF & 0.9069 & 0.9052 & 0.8966 & 0.9117 & 0.3822 & 0.1801 & 0.1126 \\
VLFS/AAKF & 0.6418 & 0.6203 & 0.6278 & 0.6296 & 0.1905 & 0.1297 & 0.0878 & 0.1413 \\
\hline
\end{tabular}




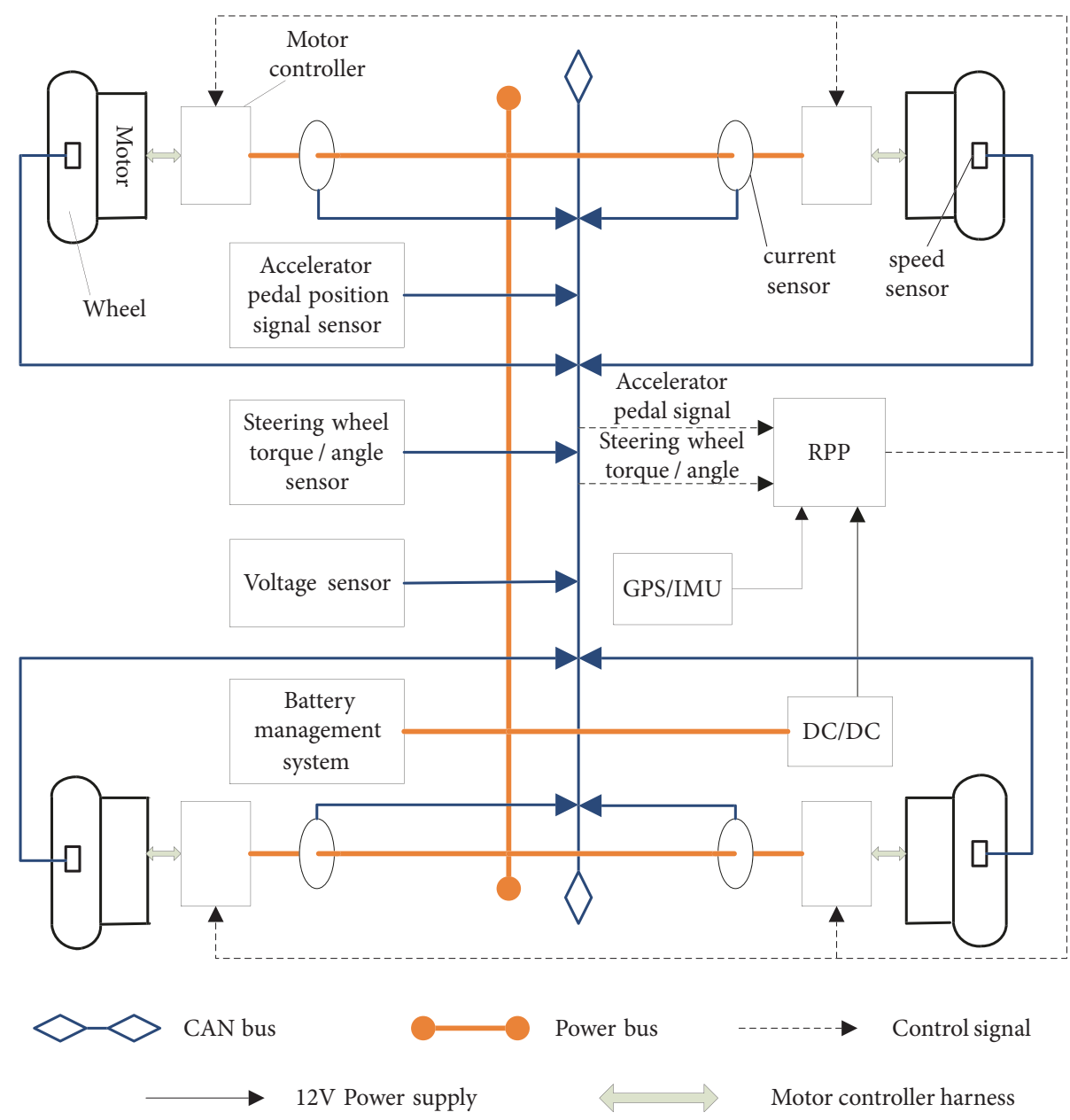

FIGURE 12: Control system and sensor network of experimental vehicle.

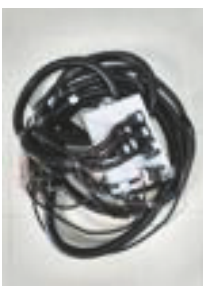

RPP

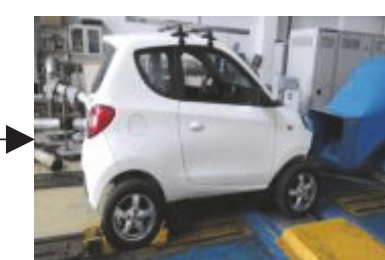

FWID-EV and chassis

dynamometer

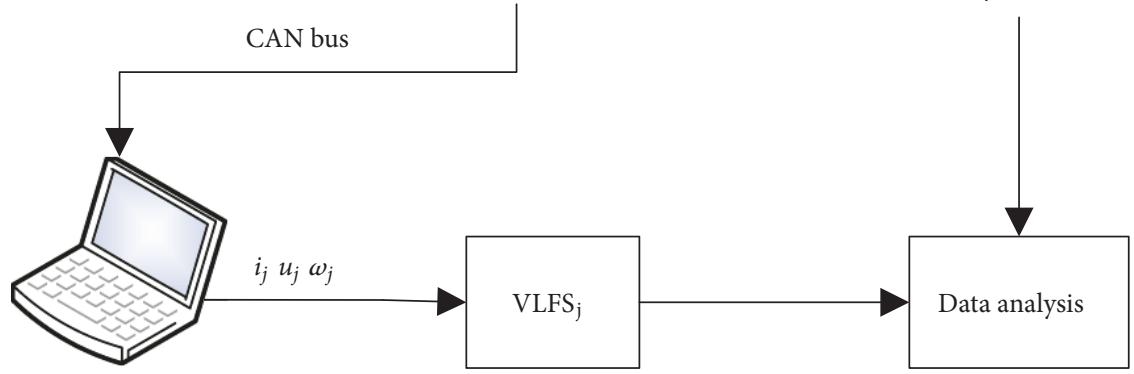

Host computer

FIGURE 13: Logical block diagram of vehicle test on chassis dynamometer bench. 


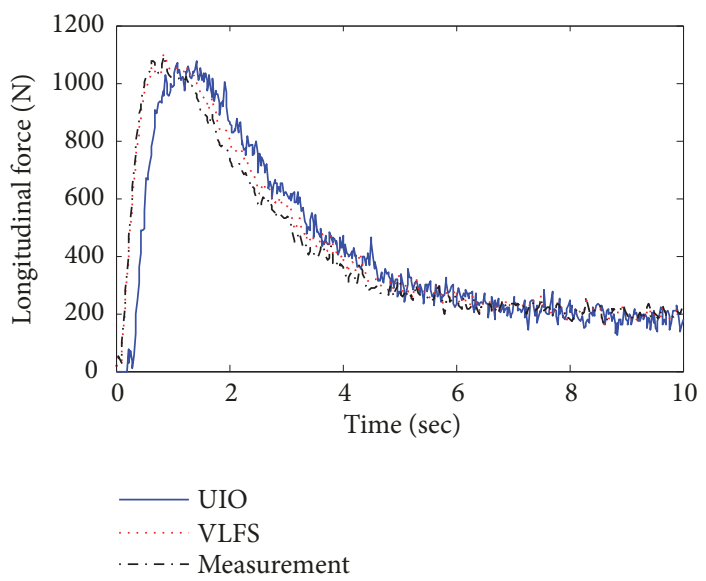

FIGURE 14: Estimation results of longitudinal force in experiment.
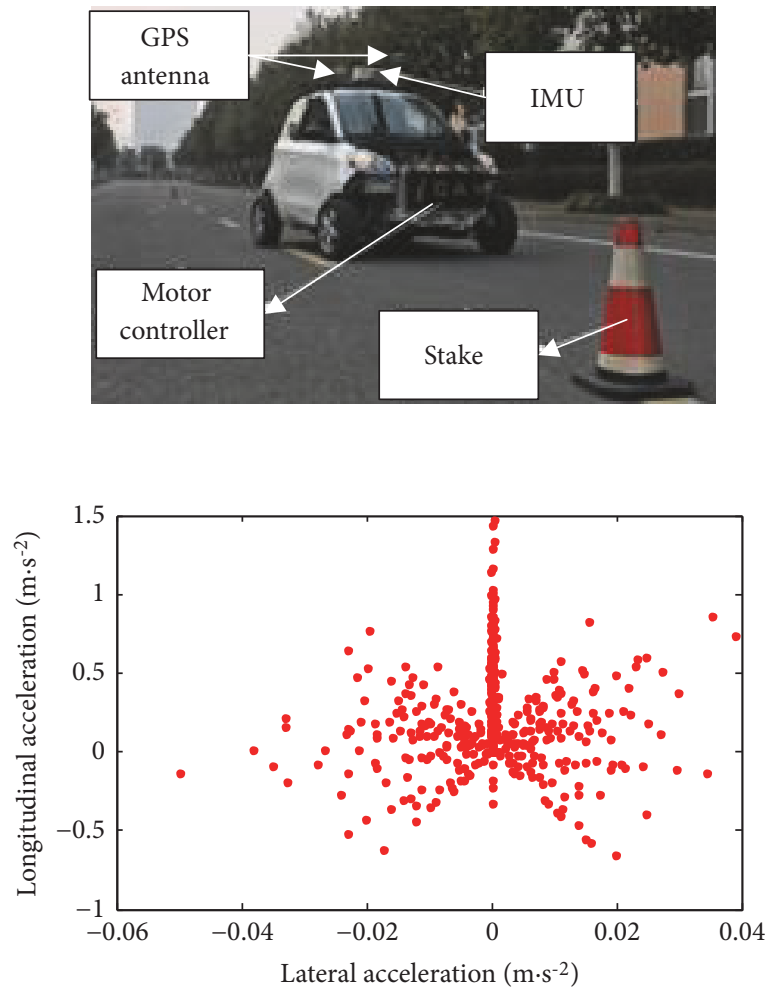

Figure 15: Road test. (a) Experimental scene, (b) G-G diagram.

TABLE 6: Comparisons of PRE in experiment.

\begin{tabular}{lccccc}
\hline PRE & $F_{x}$ & $v_{x}$ & $v_{y}$ & $\gamma$ & $\beta$ \\
\hline UIO/EKF & 372.3298 & 0.5776 & 0.0323 & 0.0259 & 0.2083 \\
VLFS/AAKF & 57.4533 & 0.2927 & 0.0113 & 0.0104 & 0.0351 \\
\hline
\end{tabular}

TABLE 7: Comparisons of ERMS in experiment.

\begin{tabular}{lccccc}
\hline ERMS & $F_{x}$ & $v_{x}$ & $v_{y}$ & $\gamma$ & $\beta$ \\
\hline UIO/EKF & 0.8009 & 0.2074 & 0.2122 & 0.1866 & 0.2088 \\
VLFS/AAKF & 0.3672 & 0.1933 & 0.1736 & 0.1601 & 0.1892 \\
\hline
\end{tabular}

\section{Acknowledgments}

This work was supported by the National Natural Science Foundation of China (Grant No. U1564201 and U1664258), the "Six Major Talent Project" of Jiangsu Province (Grant No. 2014-JXQC-004), the "333 Project" of Jiangsu Province (Grant No. BRA2016445), the Primary Research \& Development Plan of Jiangsu Province (Grant No. BE 2017129), the Natural Science Foundation of Jiangsu Province (Grant No. BK 20160525), and the Natural Science Research Projects in Colleges and Universities of Anhui Province (Grant No. KJ2016B11 and KJ2017B11). 

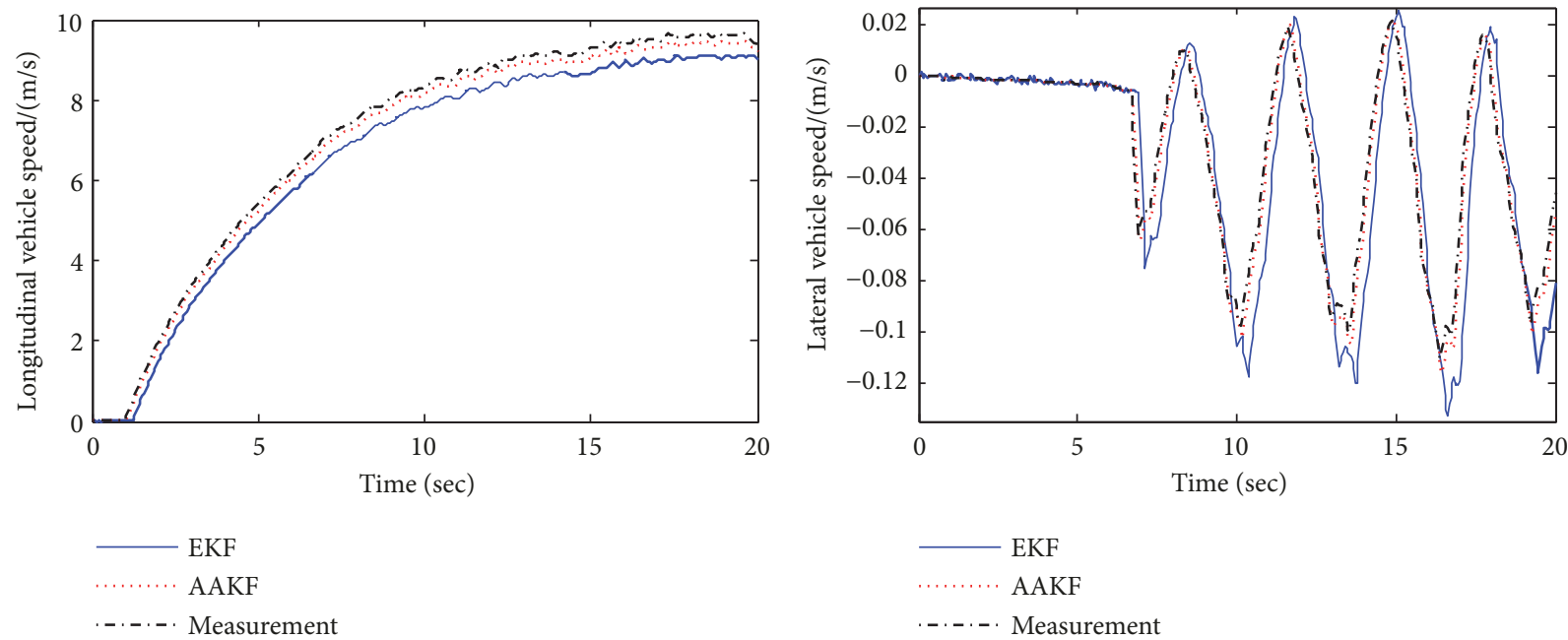

(a)
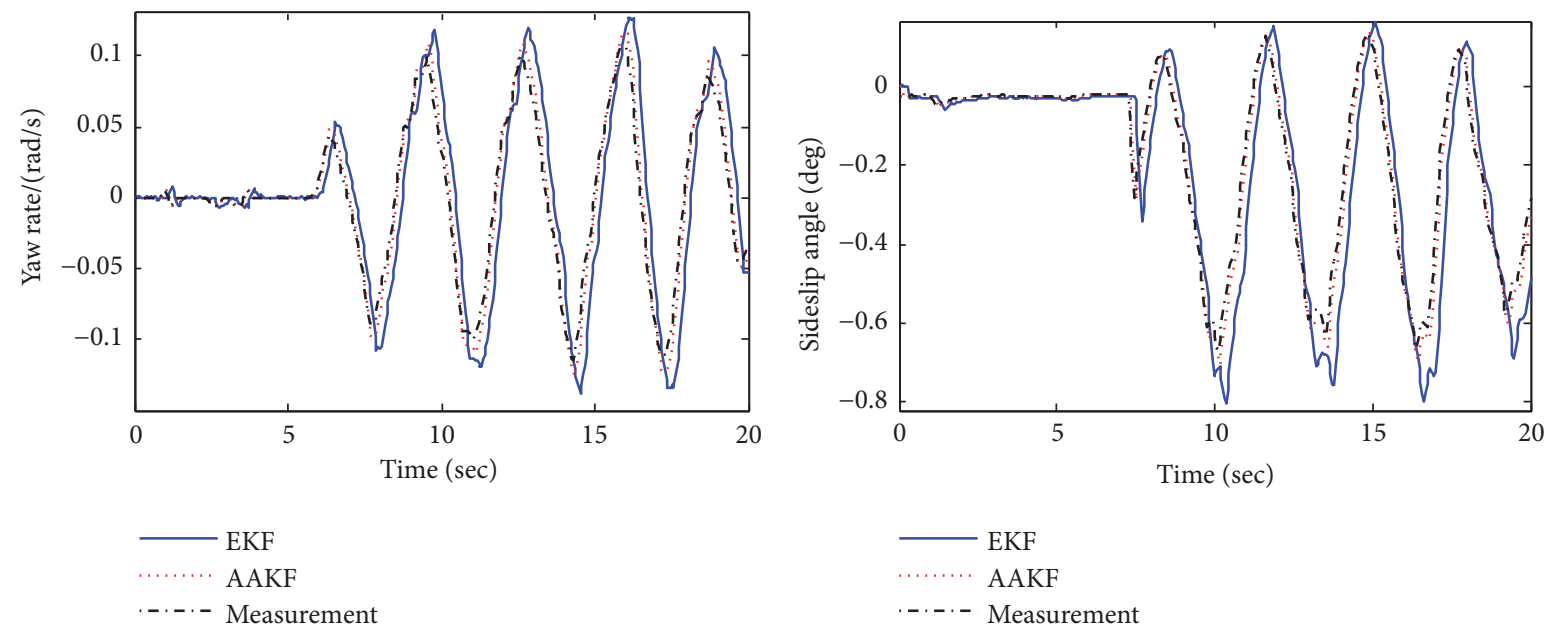

(c)

(d)

FIGURE 16: Estimation results of vehicle running states in experiment. (a) Longitudinal vehicle speed, (b) lateral vehicle speed, (c) yaw rate, and (d) vehicle sideslip angle.

\section{References}

[1] R. Wang, C. Hu, Z. Wang, F. Yan, and N. Chen, "Integrated optimal dynamics control of 4WD4WS electric ground vehicle with tire-road frictional coefficient estimation," Mechanical Systems and Signal Processing, vol. 60, pp. 727-741, 2015.

[2] R. Wang, H. Zhang, and J. Wang, "Linear parameter-varying controller design for four-wheel independently actuated electric ground vehicles with active steering systems," IEEE Transactions on Control Systems Technology, vol. 22, no. 4, pp. 1281-1296, 2014.

[3] C. Jo, J. Ko, H. Yeo, and H. Kim, "Cooperative regenerative braking control algorithm for an automatic-transmission-based hybrid electric vehicle during a downshift," Proceedings of the Institution of Mechanical Engineers, Part D: Journal of Automobile Engineering, vol. 226, no. 4, pp. 457-467, 2012.

[4] T. Chen, X. Xu, Y. Li, W. Wang, and L. Chen, "Speed-dependent coordinated control of differential and assisted steering for inwheel motor driven electric vehicles," Proceedings of the Institution of Mechanical Engineers, Part D: Journal of Automobile Engineering, vol. 232, no. 9, pp. 1206-1220, 2018.
[5] T. Chen, X. Xu, L. Chen, H. Jiang, and Y. Cai, "Estimation of longitudinal force, lateral vehicle speed and yaw rate for fourwheel independent driven electric vehicles," Mechanical Systems and Signal Processing, vol. 101, pp. 377-388, 2018.

[6] T. Chen, L. Chen, X. Xu et al., "Estimation of longitudinal force and sideslip angle for intelligent four-Wheel independent drive electric vehicles by observer iteration and information fusion," Sensors, vol. 18, no. 4, pp. 1-24, 2018.

[7] X. J. Jin, G. Yin, and N. Chen, "Gain-scheduled robust control for lateral stability of four-wheel-independent-drive electric vehicles via linear parameter-varying technique," Mechatronics, vol. 30, pp. 286-296, 2015.

[8] R. Wang, H. Zhang, J. Wang, F. Yan, and N. Chen, "Robust lateral motion control of four-wheel independently actuated electric vehicles with tire force saturation consideration," Journal of The Franklin Institute, vol. 352, no. 2, pp. 645-668, 2015.

[9] K. Nam, H. Fujimoto, and Y. Hori, "Lateral stability control of in-wheel-motor-driven electric vehicles based on sideslip angle estimation using lateral tire force sensors," IEEE Transactions on Vehicular Technology, vol. 61, no. 5, pp. 1972-1985, 2012. 
[10] R. R. Wang, H. Jing, C. Hu, F. J. Yan, and N. Chen, "Robust Ho path following control for autonomous ground vehicles with delay and date dropout," IEEE Transactions on Intelligent Transport Systems, vol. 17, no. 7, pp. 2042-2049, 2016.

[11] C. Hu, R. Wang, F. Yan, and N. Chen, "Output constraint control on path following of four-wheel independently actuated autonomous ground vehicles," IEEE Transactions on Vehicular Technology, vol. 65, no. 6, pp. 4033-4043, 2016.

[12] R. Wang, C. Hu, F. Yan, and M. Chadli, "Composite nonlinear feedback control for path following of four-wheel independently actuated autonomous ground vehicles," IEEE Transactions on Intelligent Transportation Systems, vol. 17, no. 7, pp. 2063-2074, 2016.

[13] T. Chen, L. Chen, X. Xu, Y. Cai, and X. Sun, "Simultaneous path following and lateral stability control of 4WD4WS autonomous electric vehicles with actuator saturation," Advances in Engineering Software, vol. 128, pp. 46-54, 2019.

[14] T. Chen, L. Chen, Y. Cai, and X. Xu, "Robust sideslip angle observer with regional stability constraint for an uncertain singular intelligent vehicle system," IET Control Theory \& Applications, vol. 12, no. 13, pp. 1802-1811, 2018.

[15] M. Doumiati, A. C. Victorino, A. Charara, and D. Lechner, "Onboard real-time estimation of vehicle lateral tire-road forces and sideslip angle," IEEE/ASME Transactions on Mechatronics, vol. 16, no. 4, pp. 601-614, 2011.

[16] X. J. Jin and G. Yin, "Estimation of lateral tire-road forces and sideslip angle for electric vehicles using interacting multiple model filter approach," Journal of The Franklin Institute, vol. 352, no. 2, pp. 686-707, 2015.

[17] S. Cheng, L. Li, and J. Chen, "Fusion algorithm design based on adaptive SCKF and integral correction for side-slip angle observation," IEEE Transactions on Industrial Electronics, vol. 65, no. 7, pp. 5754-5763, 2018.

[18] L. Li, J. Song, H. Li, and X. Zhang, "A variable structure adaptive extended Kalman filter for vehicle slip angle estimation," International Journal of Vehicle Design, vol. 56, no. 1-4, pp. 161-185, 2011.

[19] B. L. Boada, M. J. L. Boada, and V. Diaz, "Vehicle side slip angle measurement based on sensor data fusion using an integrated ANFIS and an Unscented Kalman Filter algorithm," Mechanical Systems and Signal Processing, vol. 72, pp. 832-845, 2016.

[20] L. Li, G. Jia, X. Ran, J. Song, and K. H. Wu, "Avariable structure extended Kalman filter for vehicle side slip angle estimation on a low friction road," Vehicle System Dynamics, vol. 52, no. 2, pp. 280-308, 2014.

[21] Y.-H. Liu, T. Li, Y.-Y. Yang, X.-W. Ji, and J. Wu, "Estimation of tire-road friction coefficient based on combined APF-IEKF and iteration algorithm," Mechanical Systems and Signal Processing, vol. 88, pp. 25-35, 2017.

[22] W. Liu, H. He, and F. Sun, "Vehicle state estimation based on minimum model error criterion combining with extended Kalman filter," Journal of The Franklin Institute, vol. 353, no. 4, pp. 834-856, 2016.

[23] K. Tin Leung, J. F. Whidborne, D. Purdy, and A. Dunoyer, "A review of ground vehicle dynamic state estimations utilising GPS/INS," Vehicle System Dynamics, vol. 49, no. 1-2, pp. 29-58, 2011.

[24] G. Baffet, A. Charara, and D. Lechner, "Estimation of vehicle sideslip, tire force and wheel cornering stiffness," Control Engineering Practice, vol. 17, no. 11, pp. 1255-1264, 2009.

[25] S. Solmaz and S. Baslamish, "A nonlinear sideslip observer design methodology for automotive vehicles based on a rational tire model," The International Journal of Advanced Manufacturing Technology, vol. 60, no. 5-8, pp. 765-775, 2012.

[26] B. Ma, Y. Liu, Y. Gao, Y. Yang, X. Ji, and Y. Bo, "Estimation of vehicle sideslip angle based on steering torque," The International Journal of Advanced Manufacturing Technology, vol. 94, no. 9-12, pp. 3229-3237, 2018.

[27] D. W. Pi, N. Chen, J. X. Wang, and B. J. Zhang, "Design and evaluation of sideslip angle observer for vehicle stability control," International Journal of Automotive Technology, vol. 12, no. 3, pp. 391-399, 2011.

[28] B. Li, H. Du, W. Li, and Y. Zhang, "Side-slip angle estimation based lateral dynamics control for omni-directional vehicles with optimal steering angle and traction/brake torque distribution," Mechatronics, vol. 30, pp. 348-362, 2015.

[29] D. M. Bevly, J. Ryu, and J. C. Gerdes, "Integrating INS sensors with GPS measurements for continuous estimation of vehicle sideslip, roll, and tire cornering stiffness," IEEE Transactions on Intelligent Transportation Systems, vol. 7, no. 4, pp. 483-493, 2006.

[30] X. Li, C.-Y. Chan, and Y. Wang, "A reliable fusion methodology for simultaneous estimation of vehicle sideslip and yaw angles," IEEE Transactions on Vehicular Technology, vol. 65, no. 6, pp. 4440-4458, 2016.

[31] A. K. Madhusudhanan, M. Corno, and E. Holweg, "Vehicle sideslip estimator using load sensing bearings," Control Engineering Practice, vol. 54, pp. 46-57, 2016.

[32] K. T. Leung, J. F. Whidborne, D. Purdy, and P. Barber, "Road vehicle state estimation using low-cost GPS/INS," Mechanical Systems and Signal Processing, vol. 25, no. 6, pp. 1988-2004, 2011.

[33] K. Nam, S. Oh, H. Fujimoto, and Y. Hori, "Estimation of sideslip angle and roll angles of electric vehicles using lateral tire force sensors through RLS and Kalman filter approaches," IEEE Transactions on Industrial Electronics, vol. 60, no. 3, pp. 988-1000, 2013.

[34] J.-H. Yoon and H. Peng, "A cost-effective sideslip estimation method using velocity measurements from two GPS receivers," IEEE Transactions on Vehicular Technology, vol. 63, no. 6, pp. 2589-2599, 2014.

[35] A. J. Tuononen, "Vehicle lateral state estimation based on measured tyre forces," Sensors, vol. 9, no. 11, pp. 8761-8775, 2009.

[36] X. Li, X. Song, and C. Chan, "Reliable vehicle sideslip angle fusion estimation using low-cost sensors," Measurement, vol. 51, no. 1, pp. 241-258, 2014.

[37] J.-H. Yoon, S. Eben Li, and C. Ahn, "Estimation of vehicle sideslip angle and tire-road friction coefficient based on magnetometer with GPS," International Journal of Automotive Technology, vol. 17, no. 3, pp. 427-435, 2016.

[38] J.-H. Yoon and H. Peng, "Robust vehicle sideslip angle estimation through a disturbance rejection filter that integrates a magnetometer with GPS," IEEE Transactions on Intelligent Transportation Systems, vol. 15, no. 1, pp. 191-204, 2014.

[39] B. J. Zhang, H. P. Du, J. Lam, N. Zhang, and W. H. Li, "A Novel observer design for simultaneous estimation of vehicle steering angle and sideslip angle," IEEE Transactions on Industrial Electronics, vol. 63, no. 7, pp. 4357-4366, 2016.

[40] H. Zhang, X. Y. Huang, J. M. Wang, and H. R. Karimi, "Robust energy-to-peak sideslip angle estimation with applications to ground vehicles," Mechatronics, vol. 30, pp. 338-347, 2015.

[41] L. Chen, T. Chen, X. Xu, Y. Cai, H. Jiang, and X. Sun, "Multiobjective coordination control strategy of distributed drive electric vehicle by orientated tire force distribution method," IEEE Access, vol. 6, pp. 69559-69574, 2018. 


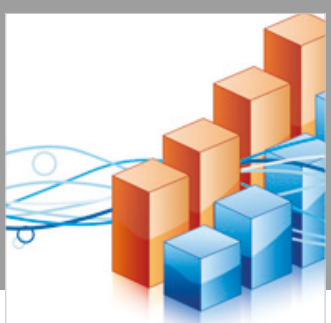

Advances in

Operations Research

\section{-n-m}
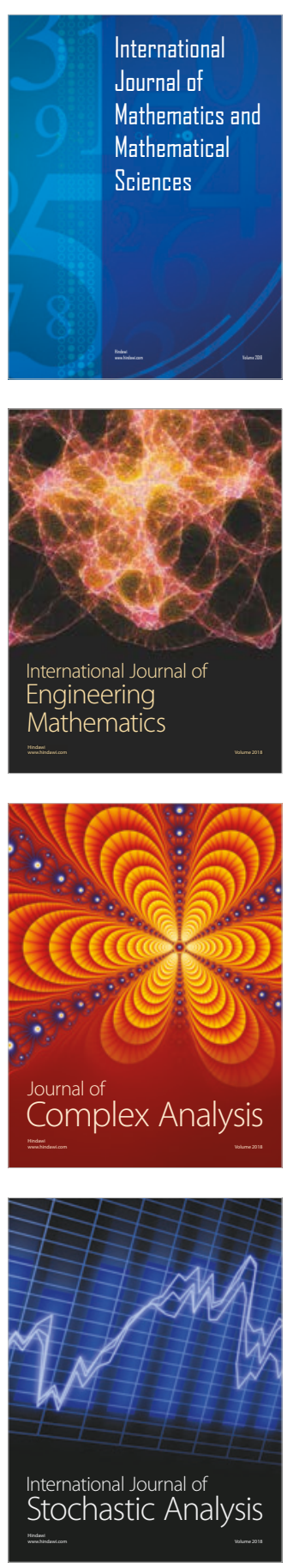
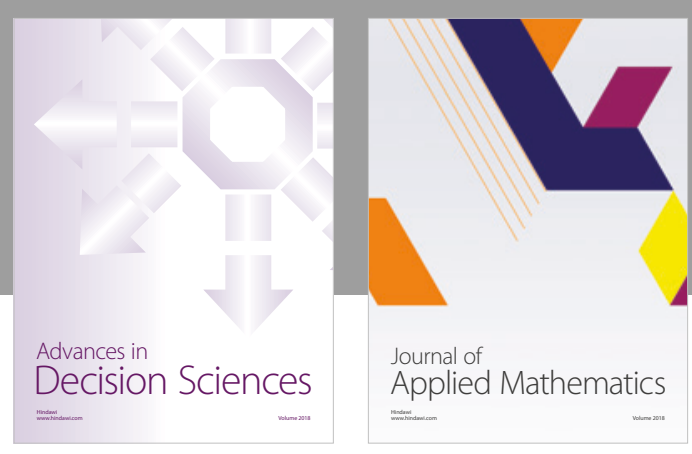

Journal of

Applied Mathematics
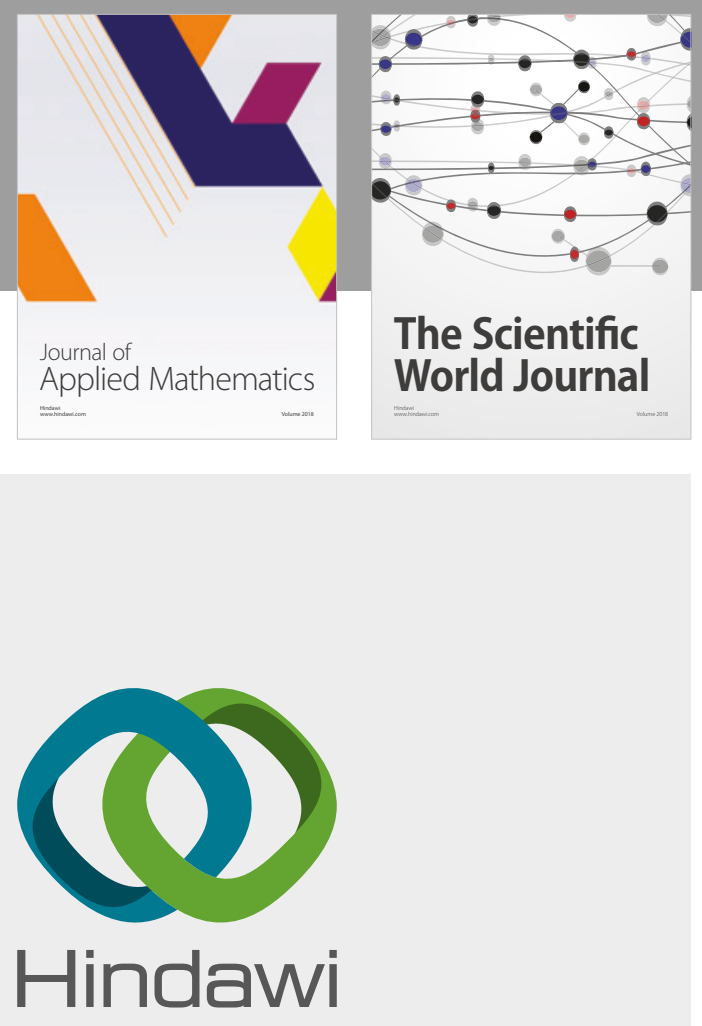

Submit your manuscripts at

www.hindawi.com

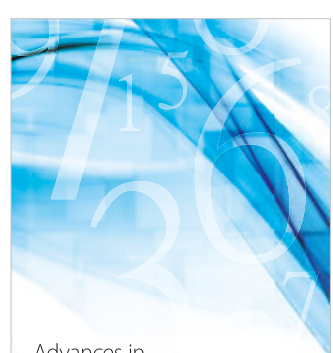

Advances in
Numerical Analysis
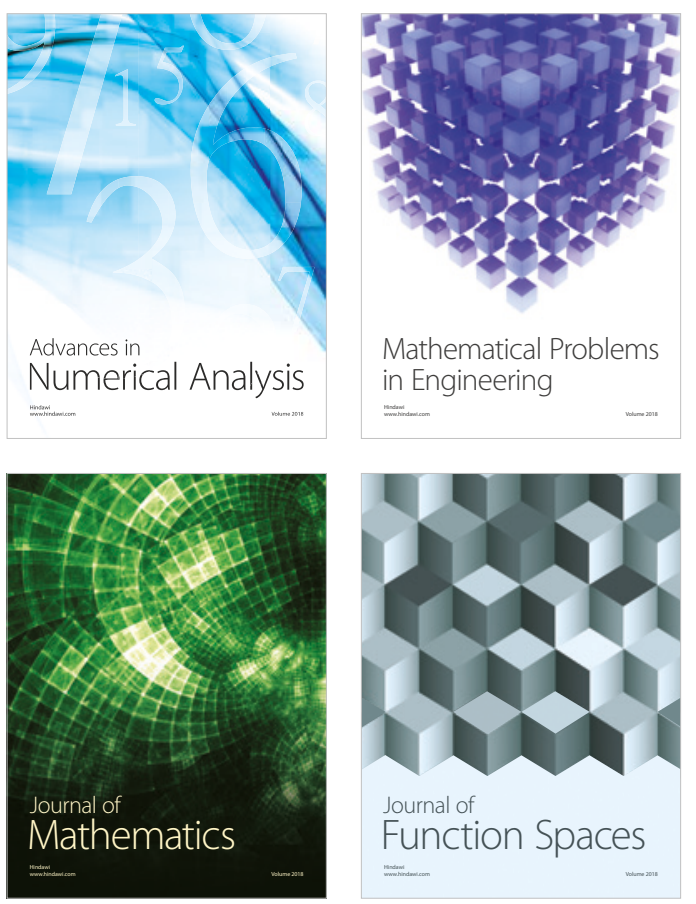

Mathematical Problems in Engineering

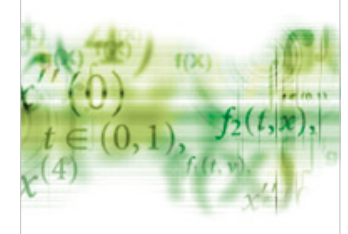

International Journal of

Differential Equations

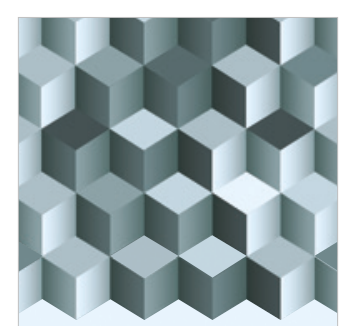

Journal of

Function Spaces

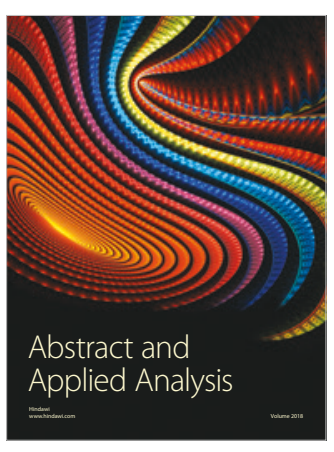

The Scientific

World Journal

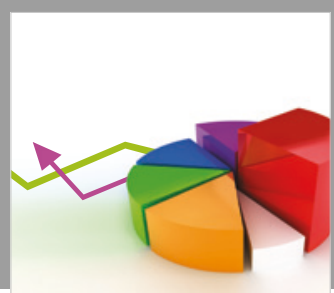

Journal of

Probability and Statistics
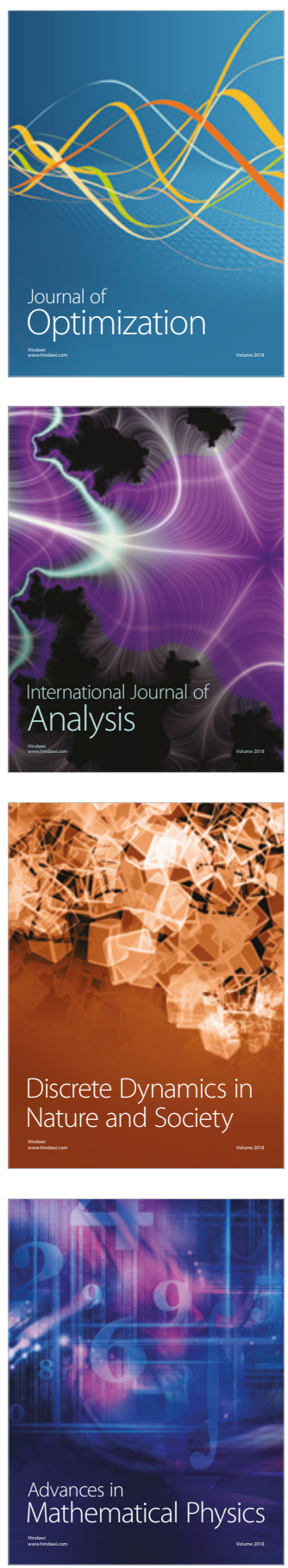